\title{
Inventory for the assessment of representational competence of vector fields
}

\author{
Stefan Küchemann $\odot,{ }^{1, *}$ Sarah Malone $\odot,{ }^{2}$ Peter Edelsbrunner, ${ }^{3}$ Andreas Lichtenberger $\odot,{ }^{4}$ \\ Elsbeth Stern, ${ }^{3}$ Ralph Schumacher, ${ }^{5}$ Roland Brünken, ${ }^{2}$ Andreas Vaterlaus, ${ }^{4}$ and Jochen Kuhn ${ }^{1}$ \\ ${ }^{1}$ Department of Physics, TU Kaiserslautern, Erwin-Schrödinger Str. 46, 67663 Kaiserslautern, Germany \\ ${ }^{2}$ Department of Education, Saarland University, Campus A 4.2., 66121 Saarbrücken, Germany \\ ${ }^{3}$ Department of Humanities, ETH Zürich, Clausiusstrasse 59, 8092 Zürich, Switzerland \\ ${ }^{4}$ Department of Physics, ETH Zürich, John-von-Neumann-Weg 9, 8093 Zürich, Switzerland \\ ${ }^{5}$ MINT-Learning Center, ETH Zürich, Clausiusstrasse 59, 8092 Zürich, Switzerland
}

(Received 11 February 2021; accepted 24 August 2021; published 1 October 2021)

\begin{abstract}
Representational competence is essential for the acquisition of conceptual understanding in physics. It enables the interpretation of diagrams, graphs, and mathematical equations, and relating these to one another as well as to observations and experimental outcomes. In this study, we present the initial validation of a newly developed cross-contextual assessment of students' competence in representing vector-field plots and field lines, the most common visualization of the concept of vector fields. The Representational Competence of Fields Inventory (RCFI) consists of ten single choice items and two items that each contain three true or false questions. The tool can be easily implemented within an online assessment. It assesses the understanding of the conventions of interpreting field lines and vector-field plots, as well as the translation between these. The intended use of the tool is both to scale students' representational competences in respect to representations of vector fields and to reveal related misconceptions (areas of difficulty). The tool was administered at three German-speaking universities in Switzerland and Germany to a total of 515 first- and third-semester students from science, technology, engineering, and mathematics subjects. In these first steps of the validation of the RCFI, we evaluated its psychometric quality via classical test theory in combination with Rasch scaling and examined its construct validity by conducting student interviews. The RCFI exhibits a good internal consistency of $\omega=0.86$, and the results of the Rasch analysis revealed that the items discriminate well among students from lower to medium-high competence levels. The RCFI revealed several misunderstandings and shortcomings, such as the confusion of the conventions for representing field lines and vector-field plots. Moreover, it showed that many students believed that field lines must not exhibit a curvature, that the lengths of field lines matter, and that field lines may have sharp corners. In its current version, the RCFI allows assessing students' competence to interpret field representations, a necessary prerequisite for learning the widespread concept of vector fields. We report on planned future adaptations of the tool, such as optimizing some of the current distractors.
\end{abstract}

DOI: 10.1103/PhysRevPhysEducRes.17.020126

\section{INTRODUCTION}

In physics education, abstract concepts and symbolicmathematical expressions form a strong contrast to concrete real-world phenomena and experimental observations. Symbolic-mathematical expressions were found to be one of the main contributors to students' difficulties in understanding physics concepts in lower and upper secondary schools [1]. One way to overcome this difficulty in understanding mathematical expressions is by the use of multiple external representations, which may help the learner to form

\footnotetext{
*s.kuechemann@physik.uni-kl.de
}

Published by the American Physical Society under the terms of the Creative Commons Attribution 4.0 International license. Further distribution of this work must maintain attribution to the author(s) and the published article's title, journal citation, and DOI. a coherent mental model of a physical concept [2]. Each of the representation types thereby offers specific advantages for conceptual understanding, and working with them may boost students' competences in interpreting and using them.

Examples of visual-graphical representations that are both closely related to phenomenological observations in experiments as well as to symbolic mathematical expressions are representations of vector fields. Vector fields are ubiquitous in nature, and they are fundamental to describe interactions of two bodies. The most prominent examples of vector fields are electric and magnetic fields. Further examples include gravitational fields or velocity fields of fluid flows such as ocean and wind currents, blood flow, or components of stress and strain in materials.

While mathematical expressions of vector fields are typical topics of introductory courses at a university, visual-graphical representations of vector fields are already 
introduced at upper secondary schools to describe fieldrelated phenomena that underlie experimental outcomes. The understanding of vector-field representations forms an integral link between experimental observation and symbolic-mathematical expressions. Eventually, to acquire an appropriate concept of vector fields, students have to understand visual-graphical representations of vector fields, and they should learn to use them.

Dealing with vector-field representations has been the focus of several recent studies. Two of them have explicitly dealt with the ability of students to use three types of representations of vector fields: algebraic notations, vectorfield plots, and field lines. Whereas Campos et al. examined the direction of translation from one representation type into another in the context of electric fields [3], Bollen et al. uncovered difficulties of students with complex vector-field representations presented detached from a physical context [4].

\section{A. Aim of this study}

Based on Bollen et al. [4], we present an approach to assess students' basic representational competence of field lines and vector-field plots. Thereby, we focus on students' ability to interpret the magnitude and directional changes in field-line representations and vector-field plots. In this work, we report essential steps in the validation of an instrument that allows the context-independent determination of specific representational competence levels of students related to these two vector-field representations. Therefore, we term the developed test Representational Competence of Fields Inventory (RCFI). Specifically, this work motivates the design of the RCFI, evaluates its psychometric characteristics in university freshmen as indicated by classical test theory and Rasch scaling, and demonstrates the construct validity of the RCFI in student interviews.

The RCFI is designed to assess the understanding of vector-field representations of middle and high school students as well as of students in university entry levels, i.e., from the moment students first encounter demonstration experiments of (for instance, magnetic and electric) vector fields until the moment when mathematical symbolic expressions of vector fields are introduced.

To make the RCFI a usable instrument for gauging representational competences in advanced placement upper secondary schools, we assessed its psychometric qualities in a sample of first-year university students of science, technology, electronics, and mathematics (STEM) subjects. The difficulties identified in this sample best show what was missed in physics classes at school. In our study, we addressed three goals: First, we assessed the psychometric quality of the RCFI through measurement theory. Second, we wanted to ascertain the extent to which specific misunderstandings and shortcomings exist by presenting certain distractors in the multiple-choice test. Among others, shortcomings concern the switch between vectorfield plots and field-line representations. Third, to get a deeper understanding of the knowledge students activate for answering the RCFI questions, interviews were conducted with a selected subsample of students with high and low scores in the RCFI. The existing test by Bollen et al. requires students to generate vector-field plots or field-line representations based on a given symbolic-mathematical expression or the respective other type of a graphical representation. While these drawing tasks are helpful for understanding various students' conceptions and missing aspects of representational competence, it is known that such generative activities pose significant challenges to students as they need to understand every aspect of the representation and be able to externalize their understanding $[5,6]$.

Furthermore, symbolic-mathematical expressions of vector fields are typically not covered in lower or upper secondary schools, so we cannot expect that the students can relate the graphical vector-field representations to a symbolic-mathematical representation. Here, the true or false and single-choice question format used in the RCFI is capable of targeting specific misconceptions of vector-field conventions, and it is easier to administer, as it also allows, for instance, relatively simple online administration. In this way, we intended that the RCFI can rate students' competence of vector-field representations in a one-dimensional assessment. So, the RCFI is meant to be an instrument that targets specific competences and elucidates students' difficulties without substantial influence of confounding factors.

\section{THEORY}

\section{A. External representations and representational competence in physics education}

Most STEM disciplines use many different types of external representations to provide information for learning or problem solving in a pedagogical or research context [7-14]. In physics, for example, several types of visualgraphical representations are often available to illustrate the same or different aspects of a concept. An instance of this is the use of field lines and vector-field plots (e.g., Refs. $[4,15])$. These two types of representations both depict vector fields in a visual-graphical way and seem quite similar on the surface. However, information about the strength and direction of the represented vector fields is coded differently in the two.

One rationale for offering different types of representations to students in physics education is that each individual representation brings its unique advantages for conceptual understanding, and its availability is assumed to affect cognitive processes during learning and problem solving in a specific way [16,17]. Fredlund, Linder, Airy, and Linder [18] refer to this as the disciplinary affordance of representations. 
Consequently, different representations provide distinct forms of access to the content as well as complementary information about the scientific concept to be acquired [2].

When students use multiple external representations (MERs) in parallel, they mentally integrate partial information from each representation to achieve thorough conceptual understanding [2,19-21]. Accordingly, a smart combination of representations even results in these MERs being worth more for scientific problem solving than the sum of the included individual representations, which is known as the collective representational affordance $[18,22]$.

What is often demonstrated, however, is that learners do not benefit optimally from external representations and MERs in terms of knowledge acquisition [23,24]. A prerequisite for learners to get the best possible results from learning with MERs is not only their immediate availability in learning materials or lessons [25] but also students' competent use of representations, which plays a crucial role in physics learning [26,27].

In this regard, however, in physics it was found that students seem to have great difficulties in the use of disciplinary representations (e.g., graph-as-picture error, $[18,28,29])$. Moreover, research indicates that the acquisition of the respective abilities might take a considerable amount of time and effort [6,30].

Fredlund et al. [18] explain these findings by the fact that representations, no matter what type, are increasingly rationalized. This means that disciplinary representations transmit information in an increasingly condensed form: small units (e.g., symbols) can contain a huge amount of information, which is also ambiguous for domain novices and can only be understood if the learners know the conventions for their interpretation and understand the relation between the representation and the concept they stand for. Kozma and Russel [31] found that science novices, in contrast to expert scientists, were not able to recognize the same principles in different representational forms and that they could also not flexibly perform transformations from one type of representation to another. Therefore, Kozma and Russel attributed to them a lack of representational competence. One can therefore assume that representational competence is an important learning prerequisite in a subject like physics, which is extremely rich in external representations.

Although there is a consensus that representational competence is central to science, and recently, the topic has also been increasingly researched [26,32], the definitions and theoretical models of this concept circulating among researchers differ to a certain extent [33]. Mostly, it is understood as a wide range of abilities [34], which are based on declarative knowledge and disciplinary conventions that can be classified as procedural knowledge [35]. In general, representational competence refers to the ability to understand, use, transfer, select, and create different domain-specific representations, such as tables, diagrams, images, and symbolic representations such as formulas and equations (e.g., Refs. [36-38]).

Based on the different cognitive processes they involve, Rau $[39,40]$ distinguishes five competences that may fall under the term representational competence. First, she defines representational sense making as the ability of learners to decode and extract relevant information from a specific representation. Second, connectional sense making is defined as the ability to recognize conceptually relevant similarities and differences between representations. As a third competence, Rau [39,40] describes representational fluency as the ability to quickly extract conceptual information from external representations. Moreover, Rau refers to connectional fluency, which allows for quick and easy translations between different representations. Metarepresentational competence as a final ability is understood as the learners' ability to reflect on their competence to deal with representations and to select appropriate representations for a specific problem, critically evaluate representations, and modify them themselves when necessary (see also Ref. [30]).

If, however, learners do not master the handling of representations but are nevertheless provided with MERs for conceptual learning, the so-called representational dilemma might arise [39]. This means that learning new concepts cannot be optimally promoted if learners must use unfamiliar external representations whose specific advantages they cannot yet make use of. Because their expertise enables them to appreciate the disciplinary affordances of domain-specific representations, school and university teachers often underestimate the hurdles such representations can present for their students' conceptual learning $[18,41,42]$.

To identify the individual educational needs of learners, representational competence must be measured appropriately. Often, qualitative methods, such as the analysis of student answers to open questions, are chosen for the assessment of representational competence [33]. Qualitative process measurements, on the other hand, are used to investigate competence development. Parnafes [43] analyzed students' gestures to infer the development of representational competence during scientific activities and found that the development of representational competence and associated conceptual understanding in physics proceeded in a coordinated way.

Until now, only a few quantitative measurements of representational competence have been developed in physics [32,44-46]. A seminal work and still widely used instrument for measuring representational competence in physics is the Test of Understanding Graphs in Kinematics (TUG-K) [44]. Apart from that, the $\mathrm{KiRC}$ is a two-tier instrument for representational competence in the field of kinematics [32]. It is designed for a standard (first year) calculus-based introductory mechanics course and comprises eleven single 
choice as well as seven multiple true or false questions involving multiple representational formats, such as graphs, pictures, and formal (mathematical) expressions (first tier). Furthermore, students express their answer confidence for selected items (second tier). To measure representational coherence in the domain of ray optics, Scheid, Müller, Hettmannsperger, and Kuhn [46] developed an instrument with fourteen items, which can be applied early in the curriculum of the lower secondary level.

The quantitative approaches to measuring representational competence presented here have in common that they do not measure representational competence independently from conceptual knowledge: The items and the included representations usually refer to a specific context in physics. However, for reasons of research theory or practical application, it may sometimes be desirable to measure certain aspects of representational competence separately from conceptual knowledge. For example, a cross-contextual instrument could be used to verify the claim that representational competence is a crucial prerequisite for acquiring conceptual knowledge [10,26]. Furthermore, universal testing instruments could be applied in different contexts, which rely on the same types of representations. Finally, context-free representational competence testing might help to tailor support for students precisely, depending on whether they lack representational competence or conceptual knowledge.

Bollen et al. [4] developed a new approach that can be used to measure representational competence in all contexts in which vector fields play a role. Their vector field representations test (VFR test) addressed students' prevalent difficulties regarding the generation of three different types of vector-field representations: formula, vector-field plot, and field lines. In an open test format, participants were required to transform given representations of vector fields into target representations by switching from one type of representation to another and explaining correspondences between the two. Since Bollen et al. aimed at separating representational competence from conceptual knowledge in future research, the items were context-free.

\section{B. Vector-field representations in physics}

Students at secondary school levels are usually first confronted with vector fields in physics courses in the context of electromagnetism. Electric and magnetic fields are both vector fields, which can be described by a magnitude and direction in every point in a subset of space. During instruction, students are typically exposed to multiple external representations of these vector fields. A way of introducing electric and magnetic fields is to start with concrete-analog representations of magnetic or electric field lines in a demonstration or hands-on experiment and then fade toward the more abstract or idealized visualgraphical representations [47,48]. For example, electric field lines can be illustrated with potassium permanganate in water [49], semolina grains in water [Fig. 1(a)], or with conductive tape [50]. Similarly, magnetic field lines can be displayed by iron filings sprinkled around the magnet (see, for instance, the magnetic field of a bar magnet [51] or a current-carrying conductor [Fig. 1(b)]).

These concrete-analog representations may then be followed by the visual-graphical field-line representations, which strongly resemble the observed field lines. In the field-line representation, continuous lines are drawn to visualize the field. At each point, the tangent to the line is along the vector at that point. Denser lines indicate a larger magnitude over a given region. Small arrow tips along the lines illustrate the direction of the field.
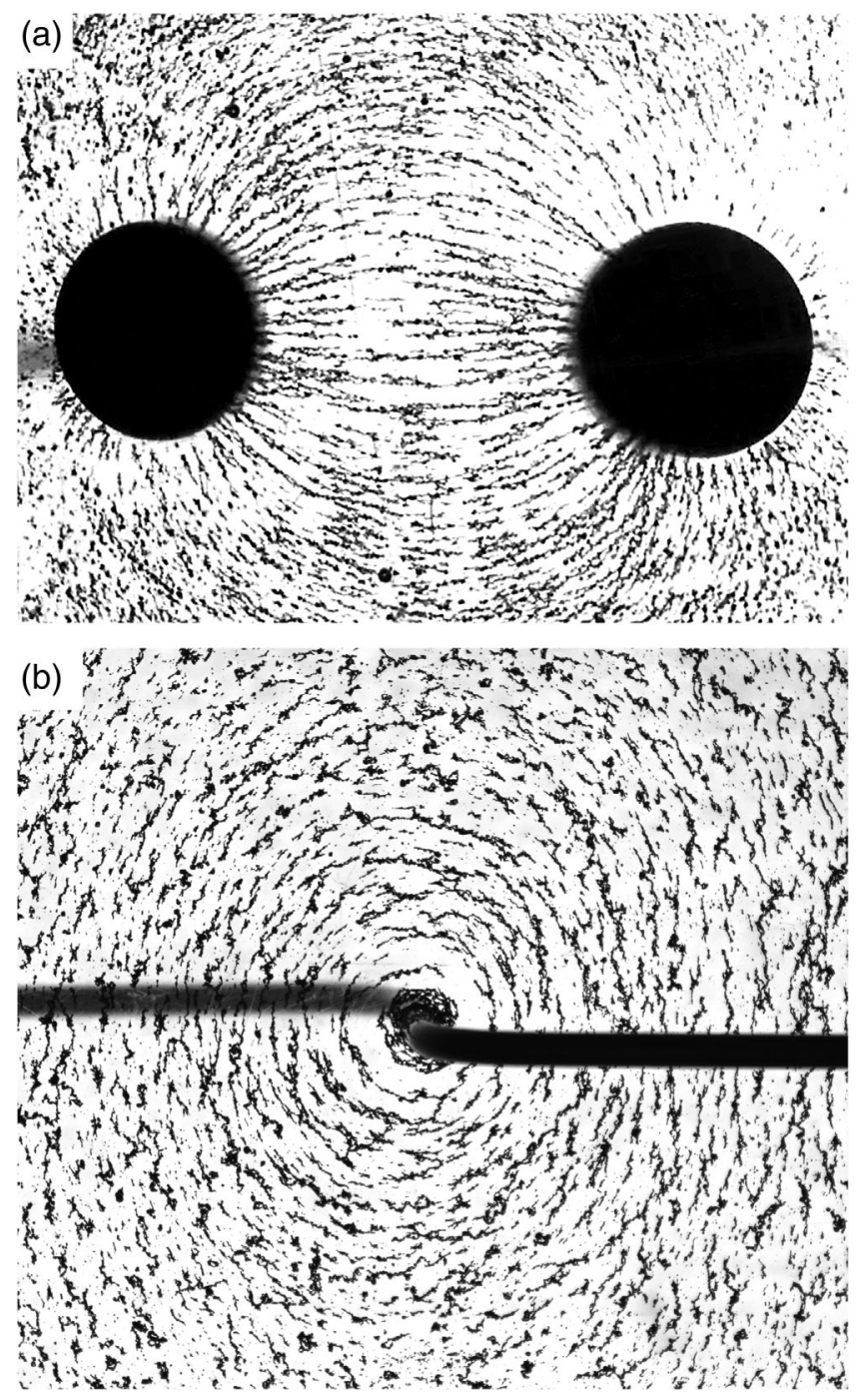

FIG. 1. Example of concrete-analog representations of vector fields. (a) A visualization of the electric field of two circular electrodes using semolina grains in water, and (b) an illustration of the circular magnetic field of a current-carrying conductor via iron filings. 
Another visual-graphical representation, which is often introduced, is the vector-field plot, also called field vector map. In the vector-field plot, a collection of arrows representing vectors of the vector field is drawn for a representative set of points. The length of each arrow describes the magnitude of the vector field, and the orientation shows the direction of the vector field at the point where the tail of the arrow is attached $[3,4]$. At last, symbolic representations in the form of mathematical functions may be used to describe the vector fields.

As previously discussed, to master the concepts of electric and magnetic fields, students should be able to make use of all representations and translate between them. Several studies have shown that this puts high demands on students' abilities [52-54].

In what follows, we shortly describe learning difficulties regarding the visual-graphical representations of vector fields. The characteristics of these representations are independent of the physics context in which the vector fields are embedded. They are also valid in purely mathematical contexts. In contrast, concrete-analog representations as described above are inherently linked to a context and therefore not suitable for the development of a universal test instrument. We also omit symbolic algebraic expressions as they are often not yet introduced at the secondary school level in Germany and Switzerland.

\section{Learning difficulties with visual-graphical representations of vector fields}

Knowledge about students' learning difficulties regarding vector-field representations is crucial to develop a test inventory. In what follows, we present typical misconceptions and difficulties described in the literature.

A difficulty with vector fields directly arises from the description given above. How do we choose a set of representative points? A vector field assigns a vector to every point in a given subset of space. This means that there is an infinite number of vectors to be drawn. Of course, this is not possible, and a selection has to be made. Gire and Price have stated that this might cause difficulties for students as they may incorrectly adjust the length of arrows so that they do not overlap or intersect [55]. This has been corroborated by Bollen et al. [4]. Besides, they have observed that students often draw only a single set of vectors, which is not sufficient to describe the magnitude and direction of the vector field over different regions.

Regarding the magnitude of the field, many students in their study did not match arrow lengths correctly. Also, students thought that arrows were centered on their location. Gire and Price found indications for a similar misconception that the vector field exists along the entire length of an arrow [55]. Moreover, Bollen et al. revealed that a majority of students have difficulties with adding vectors [4].
Considering the field-line representation, Bollen et al. also observed difficulties regarding the magnitude and the direction of the vector field [4]. Students did not match the density of field lines or simply forgot to add a direction. Indeed, a very difficult task that is inherent to the field-line representation is the addition of vector fields. While vectors can be simply added at every point in the vector-field plot, field lines cannot. The addition of two vector fields may result in a new field-line structure. Törnqvist et al. have concluded in their studies that many university students have difficulties assigning correct properties to field lines [56]. They often attached too much reality to the field lines and treated them as isolated entities. For example, $85 \%$ of second-year students did not react to crossing field lines. From interviews, they inferred that also switching between vector-field plots and field lines causes problems. Bollen et al. also found hints that students mix up the two representations. The density of vectors in vector-field plots was used to describe that magnitude and vectors were bent, while field lines were allowed to touch and cross [4]. Moreover, field lines were not tangent (e.g., perpendicular) to field vectors.

\section{INSTRUMENT DEVELOPMENT, CONTENT, AND FORMAT}

\section{A. Instrument development}

The RCFI was designed to test the students' competence to interpret vector-field plots and field-line representations. Specifically, we intended to investigate their ability to interpret the direction and magnitude of vector fields as well as the competence to superimpose two vector fields and transform the information from one vector-field representation to the other one.

The test was developed by a group of experts involving physics education researchers, physics teachers, and educational psychologists, and the test went through several iterations among these experts to ensure content validity. In the first step of the development, we performed a literature review in which we intended to identify existing tests that may be suitable for our needs. Here, we found the previous work by Bollen et al. that assesses representational competence of vector fields [4]. We used the findings of the authors (see above) to decide on content for the items and to develop appropriate distractors (see Appendix A). However, Bollen et al.'s VFR test put high demands even on the representational competence of advanced physics students. As we intended to differentiate at a lower level of competence (e.g., in the context of school education), in comparison to Bollen et al., we decided to use less complex vector fields and operations with them and also included items on the understanding of single types of vector-field representations instead of having the students always switch between representations. 
A generic strategy adapted to the target group was followed in the development of the test items. Of the possible abilities that make up representational competence [39,40], two basic ones were selected, namely, representational sense making, which addresses the ability to interpret a certain type of representation, and connectional sense making, which allows students to distinguish between different types of representations and how they depict information. Two other subcompetencies according to Rau, representational fluency and connectional fluency are associated with progressive automation in the use of representations and MERs. The fifth subcompetency, metarepresentational competence, requires a comprehensive repertoire of representation types and a good overview of these for a given context. Because of the focus of the RCFI, these higher-level subcompetencies were not addressed in this test.

\section{B. Content}

Table I shows the topic and the representation of each item. In items 1 and 2, the students are asked to determine the magnitude either of a homogeneous vector-field plot and of a homogeneous field-line representation, respectively. In items 3 and 5, it is necessary to relate the direction of a field line in a single point to the corresponding vector (item 3) or vice versa (item 5). In items 4 and 6, the students are required to translate the direction of an entire vector field from a vector-field plot to a field-line representation (item 4) or vice versa (item 6). In items 7 and 8, students need to relate the magnitude of an entire vector field in the field-line representation to a vector-field plot (item 7) or vice versa (item 8). Item 9 and item 10 contain two representations of each type in the item stem, and the task is to superimpose these two vector-field representations. Specifically, each of the two items displays two antiparallel homogeneous vector fields with different magnitudes to be superimposed, and therefore, these questions mainly address the understanding of the magnitude of vector fields represented via field lines (item 9) and vector-field plots (item 10). In items 11 and 12 , it is also necessary to superimpose two field-line representations (item 11) and

TABLE I. Overview of the items of the RCFI. The column labeled representation indicates the representation that is addressed in this item. The column labeled students' difficulties refers to the difficulties of students $([4,56])$ that are addressed in the respective item. The letter in parenthesis refers to the respective distractor. FL $\rightarrow$ VF means that the task required the translation from a field-line (FL) representation to a vector-field (VF) plot.

\begin{tabular}{|c|c|c|c|c|}
\hline Item & Format & Topic & Representation & Students' difficulties \\
\hline 1 & $\mathrm{SC}$ & Magnitude of a field & VF & Density represents magnitude as well (d) \\
\hline 2 & $\mathrm{SC}$ & Magnitude of a field & FL & Incorrect determination of the magnitude of FLs $(a, b, d)$ \\
\hline 3 & $\mathrm{SC}$ & Direction at a single point & $\mathrm{FL} \rightarrow \mathrm{VF}$ & $\begin{array}{l}\text { Confusion of FLs and equipotential lines }(\mathrm{a}, \mathrm{d}) \& \text { vectors } \\
\text { in VFs are not the tangent to FLs }(\mathrm{b}, \mathrm{c})\end{array}$ \\
\hline 4 & MTF & Direction of a field & $\mathrm{VF} \rightarrow \mathrm{FL}$ & $\begin{array}{l}\text { FLs making sharp bend (b) \& FLs are not tangent } \\
\text { to field vectors (c) }\end{array}$ \\
\hline 5 & MTF & Direction at a single point & $\mathrm{VF} \rightarrow \mathrm{FL}$ & $\begin{array}{l}\text { Vectors in VFs are not the tangent to FLs (c) \& } \\
\text { FLs must not bend (b) \& preference of homogeneous } \\
\text { solution (b) }\end{array}$ \\
\hline 6 & $\mathrm{SC}$ & Direction of a field & $\mathrm{FL} \rightarrow \mathrm{VF}$ & $\begin{array}{l}\text { Vectors in VFs are not the tangent to FLs (a, e, f) \& } \\
\text { Confusion of FLs and equipotential lines (b) }\end{array}$ \\
\hline 7 & $\mathrm{SC}$ & Magnitude of a field & $\mathrm{FL} \rightarrow \mathrm{VF}$ & $\begin{array}{l}\text { Field vectors are not tangent (e.g. perpendicular) to } \\
\text { FLs (a, e) \& confusion of FLs and equipotential } \\
\text { lines (e) \& density represents magnitude as well (c) }\end{array}$ \\
\hline 8 & $\mathrm{SC}$ & Magnitude of a field & $\mathrm{VF} \rightarrow \mathrm{FL}$ & $\begin{array}{l}\text { Preference of homogeneous solution (a) \& FLs are } \\
\text { not tangent to field vectors (c, d) \& lines do not } \\
\text { start or end at correct locations (c) \& density does } \\
\text { not represent magnitude correctly (e) \& length of } \\
\text { FLs matter (f) \& lines do not start or end at } \\
\text { correct locations (f) }\end{array}$ \\
\hline 9 & $\mathrm{SC}$ & $\begin{array}{l}\text { Magnitude after superposition } \\
\text { of two vector fields }\end{array}$ & FL & $\begin{array}{l}\text { Incomplete direction or magnitude (a, d, e) \& density } \\
\text { does not represent magnitude correctly (b) \& } \\
\text { field is zero in between lines (f) }\end{array}$ \\
\hline 10 & $\mathrm{SC}$ & $\begin{array}{l}\text { Magnitude after superposition } \\
\text { of two vector fields }\end{array}$ & VF & $\begin{array}{l}\text { Difficulties related to addition of vectors }(b-e, g) \& \\
\text { arrow length does not match magnitude of the field } \\
\text { (c, e) \& field is zero in between vectors (a) }\end{array}$ \\
\hline 11 & $\mathrm{SC}$ & $\begin{array}{l}\text { Direction \& magnitude after } \\
\text { superposition of two vector fields }\end{array}$ & FL & $\begin{array}{l}\text { Lines do not start or end at correct locations }(a, b, d) \& \\
\text { incomplete direction or magnitude }(a, b, d, c, f, g)\end{array}$ \\
\hline 12 & $\mathrm{SC}$ & $\begin{array}{l}\text { Direction \& magnitude after } \\
\text { superposition of two vector fields }\end{array}$ & VF & $\begin{array}{l}\text { Difficulties related to addition of vectors }(b, d, e) \& \\
\text { incomplete direction or magnitude }(b, c, d, e, f, g)\end{array}$ \\
\hline
\end{tabular}


TABLE II. Overview of the items that belong to each representation or translation between representations.

\begin{tabular}{lrcc}
\hline \hline $\mathrm{VF}$ & $\mathrm{FL}$ & $\mathrm{VF} \rightarrow \mathrm{FL}$ & $\mathrm{FL} \rightarrow \mathrm{VF}$ \\
\hline 1 & 2 & 4 & 3 \\
10 & 9 & 5 & 6 \\
12 & 11 & 8 & 7 \\
\hline \hline
\end{tabular}

two vector-field plots (item 12), but in this case, one of the two vector fields is a circular vector field, and therefore, it is required to understand the influence of the superposition on the magnitude and the direction. The details of the design of each distractor can be found in the Appendix A.

Table II illustrates that the RCFI has a balanced design of items addressing either conventions of vector-field plots, of vector-field lines (representational sense making), or the translation between the two representations (connectional sense making).

\section{Format}

The RCFI consists of ten single-choice questions and six true or false questions. The latter was included because previous research showed that students prefer this format, and it was found to be more reliable in comparison to complex multiple-choice questions [57,58]. The singlechoice item format was used because the distractors allow the identification of specific misconceptions and missing competences, which were also identified in prior [44] and current work.

Furthermore, we use a combination of a single-choice item format and multiple true or false questions because both types of items are easy to administer and to evaluate. The combination of single-choice and multiple true or false questions was also used in a previous assessment [32]. In some cases, like when one representation type needs to be assigned to another one, the multiple true or false format allows the identification of more than one difficulty of students and therefore offers a more detailed picture of students' thinking [59]. In other cases, either if students are supposed to make comparisons among options or the options are mutually exclusive, we selected the single-choice question format following the suggestion by Couch et al. [59]. Examples of such questions are the identification of the correct superimposed vector-field representation or the selection of the vector-field representation with the highest magnitude. Apart from that, in comparison to open-response items, multiple true-false items are able to reveal specific students' misconceptions which potentially would not be articulated in openresponse questions [60].

The single-choice questions consist of a text-based item stem partially including a visual graphical representation of a vector field in case this vector field needed to be transformed or superimposed. The number of distractors in the single choice items was varied between three and six to be sensitive to previously reported misconceptions and according to the plausibility of the distractors. The multiple true or false items consist of a text-based item stem including a visual-graphical representation of a vector or a vector field and several exemplary cases. The students are asked to decide for each one whether the given representation corresponds to the one in the item stem.

\section{METHODS}

\section{A. Test administration}

The RCFI was one element of a multipart study, which was administered in an online format via the survey software Unipark [61]. This approach had been decided upon for the present research, and due to an acute pandemic situation (COVID-19) in Germany and Switzerland, university courses were mainly conducted online in the year 2020. In September and October (beginning of the lecture period), students attending four different undergraduate university lectures in two German and one Swiss university were invited to participate in the study. All four lectures were online courses including synchronous learning phases that differed a little regarding their organization. Therefore, the respective lecturers proceeded in a different way to motivate the students to participate in the study, which was anonymous, voluntary, and not financially recompensed.

In the Lecture on Basics of Educational Research for Teacher Education (subsample 1, Saarland University, Germany) and the undergraduate course Experimental Physics 1 for Engineers (subsample 2, TU Kaiserslautern, Germany), all students were sent a link to the study at the beginning of a regular online lesson, and they could participate synchronously during the usual lecture hours. In the other two lectures (Physics for Agricultural Sciences, Earth Sciences, Environmental Science, and Food Sciences, subsample 3, and Basic Lecture for Physics Students, subsample 4, ETH Zurich, Switzerland), the participation in the study was introduced as an additional voluntary activity as a part of the usual homework. In Switzerland, the students were allowed to participate in the study for two weeks. Afterward, the survey was closed, and the survey topic was dealt with in the next lecture. The students also received general feedback on their performance in the test.

As already mentioned, the RCFI was only a part of the survey presented to the students. The overall study was conducted to set up a research project for the instructional support of learners in the field of electromagnetism. Following the link to the online survey, the students saw an information page that explained the objectives of the present research. Afterward, the students were informed about the anonymity and processing of their data and could give their online consent to participate. The RCFI was the first part of the survey. It was followed by a short concept 
TABLE III. Information of subsamples.

\begin{tabular}{|c|c|c|c|c|}
\hline & $\begin{array}{l}\text { Subsample } 1 \\
(N=188)\end{array}$ & $\begin{array}{l}\text { Subsample } 2 \\
(N=149)\end{array}$ & $\begin{array}{c}\text { Subsample } 3 \\
(N=98)\end{array}$ & $\begin{array}{c}\text { Subsample } 4 \\
(N=80)\end{array}$ \\
\hline Gender (percentage $m / f / d)^{\mathrm{a}}$ & $29 / 71 / 0$ & $85 / 14 / 1$ & $28 / 69 / 3$ & $67 / 29 / 3$ \\
\hline Mean age (SD) & $20.75(3.82)$ & $20.72(2.36)$ & $21.10(1.60)$ & $19.74(2.96)$ \\
\hline Years of physics at school (SD) & $4.45(1.69)$ & $5.57(1.90)$ & $3.11(1.27)$ & $4.08(1.48)$ \\
\hline $\begin{array}{l}\text { Mean number of study } \\
\text { semesters (SD) }\end{array}$ & $1.19(.97)$ & $1.58(1.38)$ & $3.12(.51)$ & $1.18(1.59)$ \\
\hline Most frequent field of study & Teacher education $(100 \%)$ & $\begin{array}{l}\text { Mechanical engineering (40\%), } \\
\text { electrical engineering }(28 \%)\end{array}$ & $\begin{array}{l}\text { Environmental } \\
\text { sciences }(57 \%)\end{array}$ & Physics $(55 \%)$ \\
\hline
\end{tabular}

${ }^{\mathrm{a}} m=$ male, $f=$ female, $d=$ diverse or not specified.

test on electromagnetism and Lorentz force (thirteen singlechoice items). This was followed by three items, which were used to determine the extent to which the students could remember experiments on these concepts that had been carried out in their physics lessons at school. Afterward, the students worked on ten items referring to their understanding of scientific models and experimental skills-exemplary for the field of electromagnetism. This was followed by questions on gender, age, school type, the extent of physics lessons attended at school, and the participant's current subject of study at university. At the end of the survey, a short text was used to inform the students about the reasons why survey data may not be valid in some cases (e.g., rushing through the items). The students were then asked to assess the quality of their data themselves by choosing between the three alternatives: "my data is okay," "I am not sure if my data is okay," and "my data is not okay." Overall, it took the students about $25 \mathrm{~min}$ to complete the questionnaire. The data we analyzed for this paper are openly accessible via a data repository [62].

In the following, the test administration for the representational competence test which is of particular interest here is described in more detail. Each of the twelve items was presented on a single page together with its alternative answers. The entire survey was user paced; i.e., students were able to click on a button to move on to the next question as soon as they felt ready. Within the test, students could also go back to previous items and revise their answers. However, once they had switched to the concept test, which was the next part of the survey, they were not allowed to go back to the RCFI.

\section{B. Sample}

The RCFI developed for this study was designed to assess high school students' representational competence concerning vector-field representations. Therefore, undergraduate students were selected as study participants, who had not had any experience with vector-field representations during their studies at a university and who were therefore assumed to still be at the same competence level concerning vectorfield plots and field lines as immediately after graduating from school. To ensure variance in representational competence, which was required for test validation, students were recruited from different vector fields of study—related or less related to physics. It was assumed that this procedure would result in a total sample of students who had physics lessons of varying length and magnitude during high school.

Over 1000 students were invited to participate in the study. Of these, 845 opened the link sent to them, and 540 participants completed the whole survey. Only complete datasets were included in the data analyses for the current paper. We also decided to exclude the data of those participants who obviously had not seriously completed the test. Accordingly, we excluded those students from data analysis who had taken less than $10 \mathrm{~min}$ to complete the entire questionnaire and those who had stated with an appropriate justification that their data was not trustworthy. The final sample consisted of $N=515$ participants. Descriptions of the subsamples recruited in the four different lectures are shown in Table III.

\section{PSYCHOMETRIC CHARACTERISTICS, AS INDICATED BY CLASSICAL TEST THEORY AND RASCH SCALING}

We examine the newly developed measure's psychometric characteristics. This encompasses classical test theory, including item difficulties and reliability estimation as indicated by internal consistency and item-test correlations, and the comparison of competences on related sets of items. Furthermore, we also examine how the measure's items perform in Rasch scaling [63], to see how the individual items map on students' competence levels, and whether the items function appropriately for scaling students' competences onto a common underlying dimension of representational competence. Eventually, this section concludes with a detailed distractor analysis, which reveals weaker and stronger distractors and may point toward difficulties of students.

\section{A. Item and test analysis according to classical test theory}

\section{Methodology}

Classical test theory is a central method to analyze test instruments. It assumes that an item score consists of two 
components: the true score and a random error [64]. In this work, we analyzed three measures of the classical test theory that were also suggested by Ding and Beichner [65]: the item difficulties $P_{j}$, discrimination indices $D_{j}$, and item-test correlation coefficients (point-biserial coefficients) $r_{j t}$. Item 4 consisted of four subitems (a)-(d) which are implemented as true or false questions. For the analysis, we did not consider item 4(d) because it shows negative values in the discrimination index $\left(D_{j}=-0.24\right)$ and the item test correlation coefficient $\left(r_{j t}=-0.09\right)$. As all of the remaining three subitems 4(a), 4(b), and 4(c) are designed to represent the ability to map the direction of a vector-field plot to a field-line representation, we integrated these items into a single trait. Only if a participant answered all three subitems correctly, item 4 was considered correct. If a participant made one mistake in one of the subitems, item 4 was considered incorrect. Item 5 was treated the same way as it also consists of three subitems (a)-(c) in a true or false format.

Additionally, we report the Velicer MAP test as a measure of unidimensionality and the commonly reported $\alpha$ as well as the more robust $\omega$ as measures of internal consistency of the entire item set [66].

\section{Results}

Table IV shows the results of item characteristics of all test items.

Difficulty index.-In Table IV, we can see that the difficulty index of all items ranges from 0.31 to 0.83 . Therefore, the test covers the suggested range of difficulty of 0.3 to 0.9 according to Ding and Beichner. It is visible that the test contains four relatively difficult items (items no. $1,4,5$, and 7 ) and two rather easy items (items no. 2 and $3)$. The average difficulty of the test is 0.53 .

TABLE IV. Item statistics: Item difficulty index $P_{j}$, item discriminatory index $D_{j}$, and item-test correlation coefficient $r_{j t}$ for each item $j$. The items with an ${ }^{*}$ indicate items which had several true or false subitems in which a field-line representation needed to be assigned to a given vector-field plot.

\begin{tabular}{lccc}
\hline \hline Item No. & $P_{j}$ & $D_{j}$ & $r_{j t}$ \\
\hline 1 & 0.36 & 0.54 & 0.52 \\
2 & 0.82 & 0.24 & 0.44 \\
3 & 0.83 & 0.13 & 0.37 \\
$4^{*}$ & 0.31 & 0.42 & 0.48 \\
$5^{*}$ & 0.33 & 0.49 & 0.53 \\
6 & 0.76 & 0.17 & 0.37 \\
7 & 0.31 & 0.58 & 0.59 \\
8 & 0.44 & 0.63 & 0.61 \\
9 & 0.55 & 0.63 & 0.61 \\
10 & 0.62 & 0.58 & 0.63 \\
11 & 0.54 & 0.31 & 0.38 \\
12 & 0.52 & 0.56 & 0.56 \\
Mean & 0.53 & 0.44 & 0.51 \\
\hline \hline
\end{tabular}

Item discriminatory index.-Overall, we found that the average discrimination index is 0.44 , which is in a satisfactory range $(>0.3)$ according to Ding and Beichner [65]. There are three items that are below this threshold, which are items 2, 3, and 6 . These three items have a high item difficulty index and therefore are prone to insufficiently discriminate between good and poor performers.

Item-test correlation.-The item-test correlation coefficients of all items are above the expected level of 0.2. This is an indication for a good item reliability and that each item is consistent with the other items in the test [65]. It is in agreement with the following analysis of dimensionality.

Dimensionality and internal consistency.-To verify the dimensionality of the test, we considered the Velicer map test indicating a single factor, which means that the test consists of items that test the same competence of students. Furthermore, the internal consistency of the entire test is $\alpha=0.86$ and also $\omega=0.86$, which are also in a good range.

Overall, we found that it is a satisfactorily reliable and discriminating test, which has a balanced distribution of difficulty levels and a good single item-test correlation.

\section{B. Rasch scaling}

\section{Methodology}

Rasch scaling was performed to examine how the difficulties of the items of the test cover students' representational competence, as indicated by a Wright map, and to examine the quality of scaling of students' abilities on a common dimension using item fit statistics. The twelve items of the measure were scaled according to the Rasch model in the software package TAM [67] in the $\mathrm{R}$ programming environment. A Wright map was drawn, on which students' abilities in representational competence as estimated by the Rasch model were mapped onto the same dimension as the items' estimated difficulties as estimated by the model [63]. This allows investigating to which degree the items cover the students' ability ranges well, indicating whether the items can differentiate between more and less able students regarding representational competence within specific ability ranges. Multiple items covering the same ability range, or a lack of items covering a specific ability range, would indicate that within those areas, some items are rather redundant or do not manage to differentiate between student levels of ability. In addition to the Wright map, item infit and outfit MNSQ statistics are estimated. These statistics inform about the degree to which the individual items function under the Rasch model's predictions, that is, whether they map well onto the scaling of a single underlying dimension of representational competence [63]. Cutoffs for these statistics have been proposed that vary according to the measure's intended purpose, with the strictest cutoffs going from 0.8 to 1.2 for high-stakes testing, and for survey research 0.6 to 1.4 [68]. 

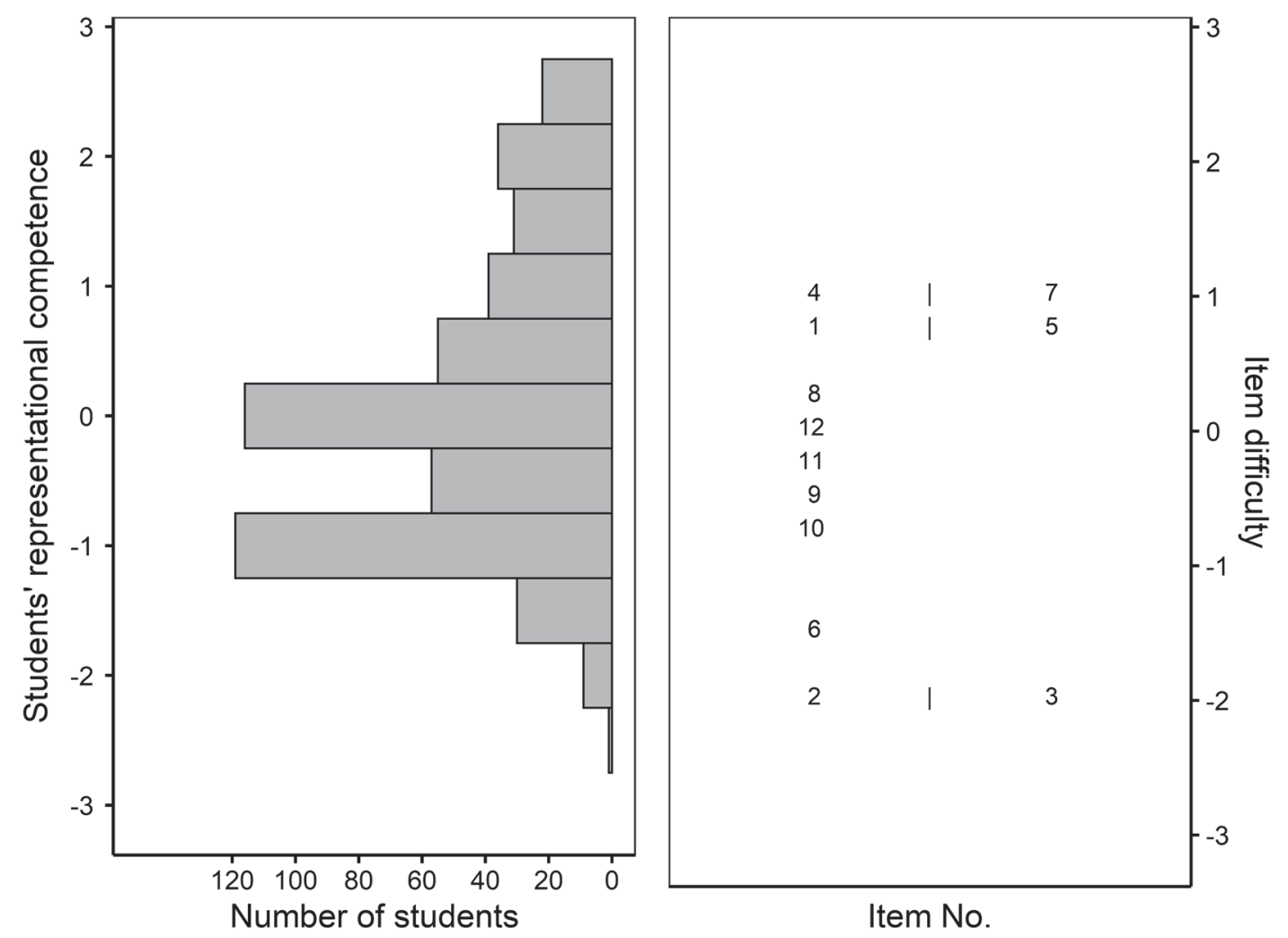

FIG. 2. A Wright map of students' representational competences and item difficulties of the RCFI.

\section{Results}

The Wright map in Fig. 2 shows students' ability ratings (higher: more able) as estimated by the Rasch model and the items' estimated difficulties (higher: more difficult) represented on the same dimension. When a student has the same ability estimate as an item, the expected solution probability is 50\%; when a student has a higher ability than an item's difficulty, the student's expected solution probability is higher, and vice versa. The Wright map shows that the three easiest items, items 2, 3, and 6, cover the abilities of the least able students well. Following the next five more difficult items (items 10, 9, 11,12, and 8), these cover the bulk of students with about-average abilities. Thus, these mentioned items overall cover the lower and average levels of student abilities well, indicating that the measure has the potential to differentiate between more and less able students regarding representational competence at these levels. The remaining four items (items 1, 5, 4 and 7) cover student abilities in the range of about 0.7 to 1.1 . Whereas these items cover a range of students with above-average representational competence, they do not cover the abilities of the ablest students (range 1.1 to 2.8). Thus, overall, the items discriminate best and cover the ability range well for university students with below-average, average, and moderately above-average abilities whereas for the assessed sample, they do not manage to discriminate well between students at the higher end of representational competence.
Table V shows the infit and outfit MNSQ statistics of all test items. The estimated statistics for all items were well within acceptable ranges, with all items apart from outfit for item 11 even reaching proposed standards for high stakes testing [68].

\section{Distractor analysis}

In this section, we show a detailed analysis of the distractors in which we evaluate the selection frequency

TABLE V. Item statistics: Item infit MNSQ (mean square) and outfit MNSQ statistics for each item $j$.

\begin{tabular}{lcc}
\hline \hline Item No. & Infit MNSQ & Outfit MNSQ \\
\hline 1 & 1.02 & 1.07 \\
2 & 1.00 & 0.86 \\
3 & 1.06 & 1.06 \\
4 & 1.05 & 1.06 \\
5 & 1.00 & 1.03 \\
6 & 1.12 & 1.18 \\
7 & 0.91 & 0.89 \\
8 & 0.92 & 0.90 \\
9 & 0.91 & 0.88 \\
10 & 0.87 & 0.82 \\
11 & 1.18 & 1.24 \\
12 & 0.99 & 0.97 \\
Mean (SD) & $1.00(0.09)$ & $1.00(0.13)$ \\
\hline \hline
\end{tabular}


TABLE VI. Relative frequency of each answer alternative. The answer alternatives marked with $\mathrm{a}^{+}$are the correct answers. Items 4 and 5 consist of three true or false questions, respectively. In this table, these two items have been coded in the way that the numbers for each answer alternative correspond to the relative frequency of participants who thought that the field-line representation in the true or false questions a, b), and c) corresponds to the vector-field plot in the item stem.

\begin{tabular}{lccccccc}
\hline \hline Item No. & $\mathrm{a}$ & $\mathrm{b}$ & $\mathrm{c}$ & $\mathrm{d}$ & $\mathrm{e}$ & $\mathrm{f}$ & $\mathrm{g}$ \\
\hline 1 & $36^{+}$ & 5 & 3 & 56 & $\ldots$ & $\ldots$ & $\ldots$ \\
2 & 6 & 5 & $83^{+}$ & 6 & $\ldots$ & $\ldots$ & $\ldots$ \\
3 & 4 & 7 & 1 & 4 & $82^{+}$ & $\ldots$ & $\ldots$ \\
4 & $74^{+}$ & 27 & 35 & $\ldots$ & $\ldots$ & $\ldots$ & $\ldots$ \\
5 & $92^{+}$ & $39^{+}$ & 25 & $\ldots$ & $\ldots$ & $\ldots$ & $\ldots$ \\
6 & 2 & 3 & 15 & $76^{+}$ & 1 & 3 & $\ldots$ \\
7 & 3 & $31^{+}$ & 60 & 3 & 3 & $\ldots$ & $\ldots$ \\
8 & 11 & $44^{+}$ & 3 & 1 & 16 & 24 & $\ldots$ \\
9 & 5 & 5 & 3 & $56^{+}$ & 1 & 26 & 3 \\
10 & 10 & 4 & 4 & 10 & 5 & $62^{+}$ & 3 \\
11 & 10 & 12 & 6 & 13 & $53^{+}$ & 1 & 5 \\
12 & $51^{+}$ & 9 & 3 & 9 & 22 & 6 & $\ldots$ \\
\hline \hline
\end{tabular}

(Table VI) and compare the distractors of items that have been designed parallel for the two types of representations or the two directions of translations.

A comparison of all the distractors of item 1 demonstrates that answer (d) is a very strong distractor with a frequency of above 50\%, which indicates that more students chose an answer with a higher density than with longer arrows. In contrast, the similarly constructed item 2 for field lines has a high frequency of correct solutions.

In items 3 and 5 , it was necessary to identify the corresponding representations of the other type in a single point. These two items specifically target the understanding of how the local direction is depicted in each representation. On the one hand, item 3 has a high solution rate, which indicates that a high number of participants correctly selects the arrow tangential to a field line. On the other hand, in item 5, it is remarkable that the field line in part (b) has a low frequency of correct solutions, which means that the assignment of a field line to a given vector at a specific point is more difficult for students.

In item 4 and item 6, it was necessary to assign an entire vector-field representation of one type to the other type. In item 4, the distractors (b) and (c) are both relatively strong distractors. In comparison, item 6 has a relatively high frequency of correct solutions and rather weak distractors. However, the comparison of these two items is not straightforward as the format between these two items is not identical, which does not allow a clear conclusion.

In item 7 and item 8, it was necessary to transfer from one vector-field representation to another one, and in these two items, there was an underlying gradient. It becomes evident that item 7 has a significantly lower frequency of a correct solution, which might be attributed to the fact that it has a very strong distractor (c) with a selection frequency of $60 \%$. This distractor exhibits a surface similarity to the fieldline representation in the item stem with an increasing density of vectors in the direction of the gradient in the field-line representation. In item 8 , the strongest distractor is answer (f), which shows that the field lines suddenly end, but the lengths of the field lines show an increase similar to the vectors representing the vector-field plot in the item stem.

In items 9 and 10, it was necessary to superimpose two homogeneous vector fields with different magnitudes pointing in opposite directions either in the field-line representation in item 9 or the vector-field plot in item 10. Although the frequency of a correct solution is only slightly higher in item 10, the distribution of distractor choices is not. In item 9, answer (f) shows an alternating vector field of field lines pointing up and down, and it is a strong distractor with $26 \%$. The parallel distractor in item 10 was only selected by $10 \%$ of the students, which in this item, is as high as the frequency of answer (d), which shows an empty vectorfield plot. In comparison, the distractor with an empty vector field in item 9 only has a frequency of $5 \%$.

In items 11 and 12, it was necessary to superimpose a homogeneous and a circular vector field either as the field-line representation (item 11) or as a vector-field plot (item 12). Here, the frequency of a correct solution was relatively similar, but the selection of distractors also shows noticeable differences. In item 12, the strongest distractor is answer (e) which shows a vector-field plot that is slightly stronger in the bottom left quarter than the correct solution. The comparable distractor (a) in item 11 only exhibits the frequency of $10 \%$, which is slightly lower than the selection frequencies of answer (b), which shows only half of the correct vector-field plot and an empty vector field on the left-hand side, and the frequency of answer (d), which is a vector field that is rotated clockwise by $90^{\circ}$ in respect to the correct solution.

\section{CONSTRUCT VALIDITY, AS INDICATED BY STUDENT INTERVIEWS}

In addition to the newly developed instrument's psychometric characteristics, we examine the construct validity through student interviews. The construct validity indicates the quality of inferences made by students during correct and incorrect answers [69]. Student interviews help to evaluate the construct validity since they show the arguments of high and low performing students. Therefore, they contain information, for instance, whether items lead students to incorrect answers despite applying correct reasoning potentially due to distracting elements, or whether students systematically arrive at correct answers with incorrect arguments.

\section{A. Methods}

After the administration of the test, we performed individual interviews with thirteen participants (all male) 
to understand the cognitive strategies and conceptions of students. The interviewer was a master's student in physics education with experience in designing and conducting interviews. The participants were all a part of subsample 2, the average age was 20.8 years, and the mean number of the study semester was 2.1. The participation was voluntary and it was compensated with 10 euros. For the interviews, we selected those nine items with the lowest difficulty index (items no. 1, 4, 5, 7, 8, 9, 10,11, and 12), because in items with a low difficulty index, we expected to receive more interesting answers that are potentially related to difficulties of students than in items with a high difficulty index. The interviews were conducted via Zoom due to prevailing contact restrictions, and they took on average eighteen minutes. After the students gave consent to the audio recording, each item was presented separately to the students via the screen-sharing app in Zoom and, after each item, the students were asked how they solved it. If the students did not consider a certain distractor during their reasoning, the interviewer asked for this distractor. The interviews were recorded with the included video recording app in Zoom. During the recording, the videos of the interviewee and the interviewer were turned off, so only the voices were recorded. The interviews were administered in German and transcribed subsequently. The included interview passages in this manuscript were translated to English, and language errors were corrected to improve readability.

\section{B. Results}

Table VII shows the selected answers of the interviewed students. It is visible that difficult items such as item 1, item 7, or item 8 also exhibit a relatively high difficulty for the interviewed students. The score of the interviewed students in these nine items range from two correct answers to nine correct answers (mean score $\langle P\rangle=6.1$; median $M_{P}=6$; standard deviation $\left.\sigma_{P}=2.4\right)$.

It is noticeable that the participants in the interviews had a slightly higher frequency of correct answers than the entire sample. The reason for this might be that the interviewer asked about distractors that were previously unmentioned. In some cases, this led students to reconsider their selection (see below).

In the following, we summarize the main arguments and the reasoning of students during the interviews. In this section, we present summaries of interviews to four items (items 1, 4, 7, and 8; see Fig. 3) because these four items showed the highest frequency of incorrect answers during the interviews. A selection of complete interviews of at least one representative correct answer and one incorrect answer for each item in Table VII are added in the Appendix C. We gave random names to the students to discriminate between them. The names in this section are identical to the ones in the Appendix C, i.e., the same name refers to the same student.
TABLE VII. The number of choices of a specific option during the interviews. There was a total number of thirteen interviews. The answer alternatives marked with $\mathrm{a}^{+}$are the correct answers. The items with an ${ }^{*}$ indicate items that had several true/false subitems. The numbers indicate how many participants said that the field-line representation of that subitem was a match with the given vector-field plot. In item no. 5, more than one field-line representation could be assigned to the given vector-field plots. The summaries of student interviews of items marked with $\mathrm{a}^{\dagger}$ are presented in this section. A selection of complete student interviews to all items in the Table can be found in the Appendix C.

\begin{tabular}{lccccccc}
\hline \hline Item no. & $\mathrm{a}$ & $\mathrm{b}$ & $\mathrm{c}$ & $\mathrm{d}$ & $\mathrm{e}$ & $\mathrm{f}$ & $\mathrm{g}$ \\
\hline $1^{\dagger}$ & $5^{+}$ & 0 & 0 & 8 & $\ldots$ & $\ldots$ & $\ldots$ \\
$4^{* \dagger}$ & $13^{+}$ & 0 & 2 & $\ldots$ & $\ldots$ & $\ldots$ & $\ldots$ \\
$5^{*}$ & $13^{+}$ & $4^{+}$ & 0 & $\ldots$ & $\ldots$ & $\ldots$ & $\ldots$ \\
$7^{\dagger}$ & 1 & $6^{+}$ & 6 & 0 & 0 & $\ldots$ & $\ldots$ \\
$8^{\dagger}$ & 1 & $8^{+}$ & 1 & 3 & 0 & 3 & $\ldots$ \\
9 & 0 & 1 & 0 & $11^{+}$ & 0 & 0 & 1 \\
10 & $12^{+}$ & 0 & 0 & 1 & 0 & 0 & 0 \\
11 & 1 & 0 & 0 & 0 & $11^{+}$ & 0 & 1 \\
12 & $12^{+}$ & 0 & 0 & 0 & 1 & 0 & $\ldots$ \\
\hline \hline
\end{tabular}

For item 1, the interviews showed that students who solved the item correctly mainly justified their solutions with the appropriate conventions regarding the representation of the magnitude in the vector-field representation. Less frequently, students spontaneously used a self-chosen context to clarify the conventions to themselves and to conclude the correct solution, e.g., in the case of Michael, who reasoned as follows: The length of the arrows, the amount, always shows how strong it is. If we had a parallelogram of forces like this, we would have it too. You can also read the strength of the force from it. In this case, it is A because the arrows are the longest. Students who solved item 1 incorrectly mostly confused the magnitude conventions for field lines and vector-field representations in their reasoning.

In item 4, students who answered correctly typically noticed that the vector-field plot has a circular shape and discarded the field line representations $\mathrm{B}$ and $\mathrm{C}$ using symmetry arguments. On one occasion, a student implied an aspect of force vectors to arrive at the correct answer. Apart from that, some students also thought that field-line representation $\mathrm{C}$ matches the given vector-field plot because it has an elliptical symmetry.

In item 7, students who solved this item correctly often used both the direction and the magnitude to arrive at the right answer. Typically, they excluded answers because of arrows pointing in the wrong direction and correctly translated from the increasing distance between the field lines in the given representation to the length of the vectors in the answer alternatives. Students who did not answer this item correctly regularly derived the answer from surface similarities: I would say $C$ because it looks most similar. Yes, and the arrows are all on the same level. Or they 
1.) Each of the figures below shows a section of a vector-field plot. Which vector field has the largest magnitude?

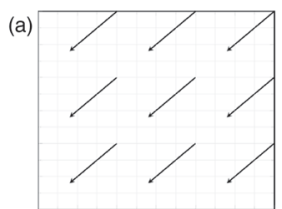

(b)
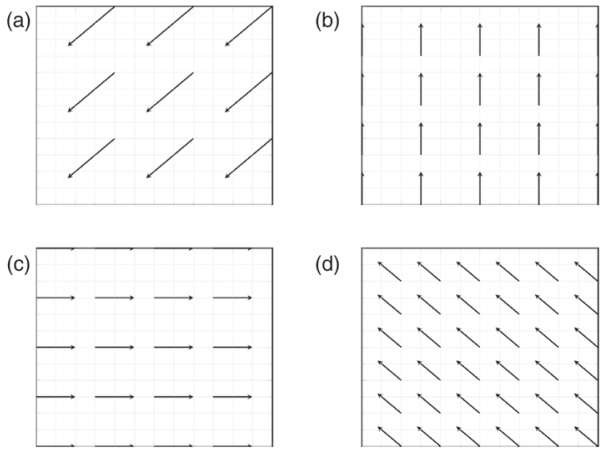

7.) The figure on the right shows the field-line representation of a field. Which vector-field plot corresponds to this field-line representation?
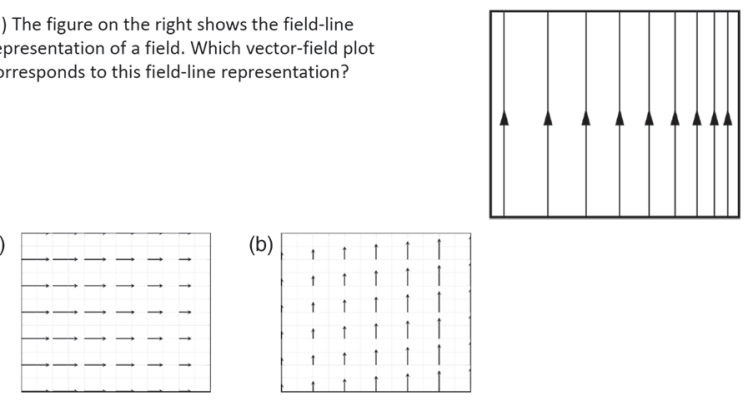

(c)

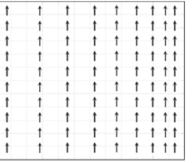

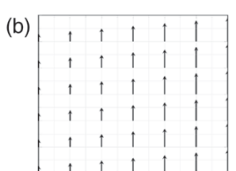

(d)

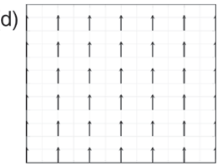

(e)

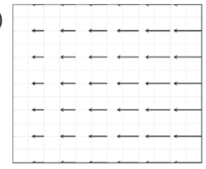

4.) The figure below shows

ector-field plot. Indicate for eac field-line representation a) to c whether it corresponds to the

given vector-field plot.

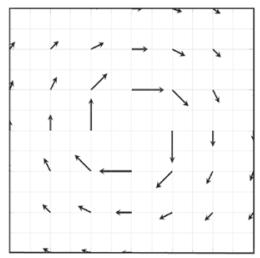

Does the field-line representation correspond to the vector-field plot?

(a)

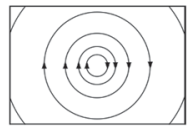

(b)

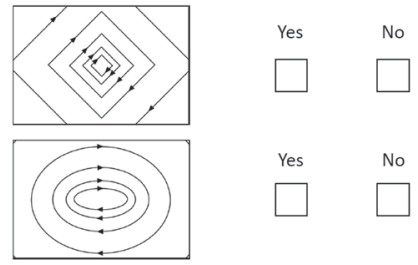

8.) The figure on the right shows the vector-field plot of a vector field. Which field-line representation corresponds to this vector-field plot?

(a)

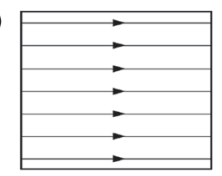

(c)

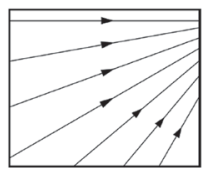

(b)

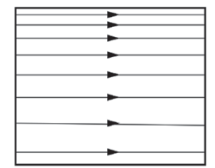

(c)

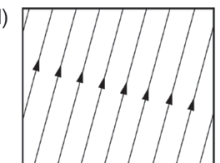

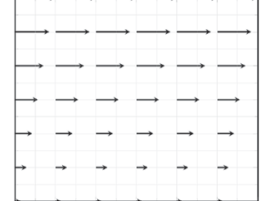

(e)

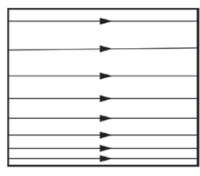

(f)

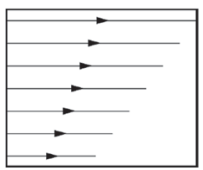

FIG. 3. Items $1,4,7$, and 8 of the RCFI.

assumed an orthogonal relationship between field lines and the vectors.

In item 8, students who chose the correct answer sometimes used directional arguments to exclude some answer options and generally related the magnitude convention of vector-field plots and field lines correctly: I would say $B$ is correct and corresponds to the vector image because the field lines are denser at the top and less dense at the bottom, which would correspond to the length of the vector arrow. Students who chose an incorrect answer typically reasoned with surface similarities and neglected the required convention of field lines: I choose $F$ because the vectors below are shorter, so the field lines below should normally be shorter.

Overall, in several cases, symmetry reasons were used to conclude the answer. Besides, the students tried to recall concrete field-line and vector-field representations that they had been exposed to before. Apart from that, students sometimes transferred the problem into a familiar context (such as force vectors in Newton's mechanics and magnetic fields). Typical errors resulted from the confusion of conventions of vector-field plots and field-line representations. Typically, for incorrect answers, students started by explaining the conventions of field-line representations and vector-field plots.

\section{OUTLOOK, AND LIMITATIONS}

\section{A. Discussion}

The present study addresses the current topic of representational competence, which is of basic importance for understanding abstract contents, such as the concept of vector fields in physics. Our goal was to develop a multiple-choice test to measure basic competences in handling visual-graphic representations of vector fields (Representational Competence of Fields Inventory). The test is designed for advanced placement classes at the secondary and high school level, therefore, the presented vector-field representations are less complex than those used in tests at the university level [4]. Moreover, we also avoided symbolic-mathematical representations used for advanced understanding $[3,18]$, but which can be expected to overstrain our target group. Besides, although representational competence is conceived as an ability with multiple subcategories [34], we limited ourselves to the basic operations with representations defined by $\operatorname{Rau}[39,40]$. At the current stage, the validation is not completed as it was not administered to parts of the target group, i.e., to students from upper secondary schools, but it is already a well suitable test to assess students' representational competence at the university entry level. 
Our test was presented to a sample of university freshmen with STEM aspirations, who had undergone physics education at school on topics related to vector fields. Still existing misunderstandings and shortcomings in this sample indicate severe failures during students' learning process, which should be directly addressed by teachers. The RCFI can be used for formative assessment to get insights into students' prior knowledge as well as in their learning trajectories during instruction. In addition, the RCFI can serve as a standardized instrument for summative assessment that allows comparing learning gains between different groups of students.

Our first goal was to assess the psychometric characteristics of the RCFI. Results revealed a rather high internal consistency. Rasch Scaling indicated satisfactory differentiation between students with rather low, average, or moderately above-average ability in representational competence. In the current sample, the differentiation within the range of highly competent students was less satisfactory. This, however, can be expected to be different when the test is applied to the sample it is meant for, which are students from upper secondary school. Results of Rasch scaling also revealed that the items are in accordance with the requirements of a one-dimensional scale, with only one item (No. 11) being an outlier. Future studies will show whether this item needs amendments.

The second goal of the study was to get a deeper insight into the knowledge students used for solving the problems. The interviews suggested that students often relied on memories of vector-field representations they remember from a specific context, as there are force, magnetic or electric fields. While this seemed to be helpful in the cases we observed in the interviews, it seems that most students are unaware of the constraints a certain context may add to the representation. For example, electric fields of two charges have a nonzero divergence, which indicates that the electric field has sources and sinks and that the field lines are not closed. In contrast, the magnetic field of a currentcarrying conductor has a zero divergence and a nonzero curl, which means that the field lines are closed and there are no sources or sinks. In sophisticated answers, students started outlining the conventions of vector-field plots and field lines and then deductively concluded on the correct answer, partially concluding on the length and direction of vectors in a vector-field plot after a pointwise subtraction or addition during the superposition of two vector fields. In this way, the arguments of correctly answering students contain expertlike reasoning approaches that confirm the construct validity of the RCFI.

In the formerly developed representational competence test of vector fields by Bollen et al., the authors observed that students experience difficulties with the addition of vector-field plots and they fail to recognize the field-line density as the magnitude of the vector field when they translate from field-line representations to vector-field plots or vice versa [4]. Also, Campos et al. observed that students have difficulties relating the density with the magnitude of field-line representations [3]. These observations guided the way we designed items, as several distractors used in our test were stimulated by their work. In addition to the students' difficulties identified by Bollen et al., we observed that students applied the field-line convention, according to which density indicates magnitude mistakenly to vector-field plots and thereby disregarded the length of the vectors as the actual measure of the magnitude. Apart from this confusion of the conventions, we also observed that students mistakenly thought that field lines cannot exhibit a curvature. A further source of misunderstanding representations was the assumption that the lengths of field lines matter, which implies that field lines may end at specific points, and the field lines can have sharp corners.

\section{B. Outlook}

The purpose of our instrument was to highlight different levels of understanding vector-field plots and field-line representations, including misunderstandings that can be used for summative as well as for formative assessment. As the reported results are important steps towards a robust instrument, the RCFI will certainly gain from further amendments resulting from applying it to additional samples. However, given the sufficient psychometric characteristics, already in its current form, it can be used as a reliable indicator of learning gains resulting from instruction. In the light of the deficiencies in the adequate understanding of representations of the physical concept of field that were even found in a sample of learners positively selected for their preferences in physics, there is a need to improve instruction and practicing in this area. It may be worthwhile to train the appropriate use of representations already when teaching electric or magnetic fields typically introduced in lower secondary school. In this context, the RCFI also still needs to prove its validity in a sample from upper secondary schools. Future research should highlight the relationship between representational competences and the conceptual understanding of the physical concept of vector fields (e.g., Refs. [2,39]). In this line, the next step would be to relate the representational competence to the understanding of vector-field concepts in various areas of physics. The fact that the abstract concept of a vector field can be represented by field lines as well as by vector-field plots is a challenge and an opportunity at the same time. As similar pictures have a different meaning in each kind of representation, confusion is inevitable. However, once learners have understood what exactly they have to keep apart when faced with the two kinds of representations, they may take advantage of each of them. Observing in detail how this learning process takes place should be a focus in future research on physics education. 


\section{Limitations}

Despite the promising possibilities that the development of the RCFI yields, there are some points to consider. The generalizability of the interview data is limited by the fact that the students were chosen from only one of the four subsamples and that they were not selected randomly for the interviews, but rather volunteered for it. Moreover, we limited ourselves to nine items in the interview; therefore, our insights into problem-solving processes are still in their infancy.

\section{ACKNOWLEDGMENT}

The authors are grateful to Sascha Sprengard for performing the physics experiments to visualize vector fields and providing the corresponding photographs in Fig. 1.

\section{APPENDIX A: DETAILS OF THE DESIGN OF EACH DISTRACTOR}

In items 1 and 2, the students are asked to determine the magnitude either of a homogeneous vector-field plot and a homogeneous field-line representation, respectively (Figs. 4 and 5). Both items have three distractors. In item 1 in the correct answer is the one with the longest arrows pointing in the direction of $225^{\circ}$ (lower-left direction) in respect to the positive $x$-axis which is aligned in horizontal direction (and which corresponds to $0^{\circ}$ ). In the distractors, we varied the direction, length, and distance between vectors. Bollen et al. found that students confuse conventions of field lines and vector-field plots. Therefore, we included one distractor in which the vectors have a small distance and a short length pointing in the (upper left) direction of $135^{\circ}$, i.e., the vectors in this distractor have a high density, which would mean in a field-line representation that this distractor exhibits the highest magnitude (answer: d; students' difficulty: density represents magnitude as well [4]). One distractor has a long vertical component and a zero horizontal component pointing upward $\left(90^{\circ}\right)$, and another distractor has the same length

1.) Each of the figures below shows a section of a vector-field plot. Which vector field has the largest magnitude? (a)

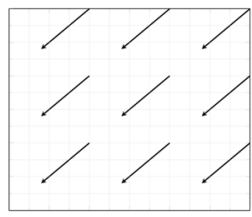

(c)

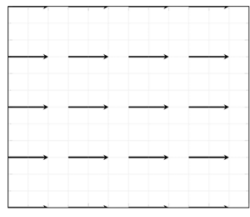

(b)

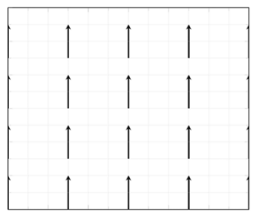

(d)

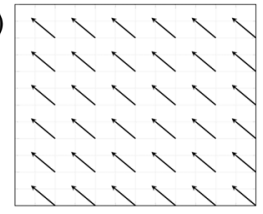

FIG. 4. Item 1.
2.) Each of the figures below shows a section of a field-line representation. Which vector field has the largest magnitude? (a)

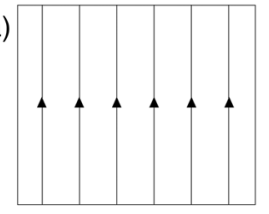

(c)

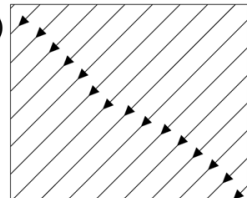

(b)

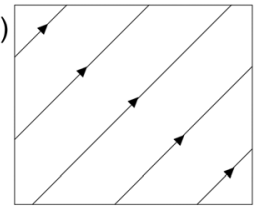

(d)

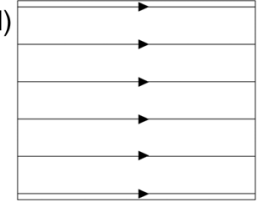

FIG. 5. Item 2.

but zero vertical component and a long horizontal component. In item 2, we included the same distractors as in item 1 , but in this item, all field lines cover the same area, i.e., we did not vary the length of the field lines. In the distractors a, $\mathrm{b}$, and d, we considered the previously reported students' difficulty of an incorrect determination of the magnitude of field lines [4].

In items 3 and 5 , it is necessary to relate the direction of a field line in a single point to the corresponding vector (item 3, Fig. 6) or vice versa (item 5, Fig. 7). This means that the answer alternatives are only single vectors. Item 3 contains a circular field-line representation in the item stem. The arrows that indicate the direction of the field lines are all indicated in the topmost point of each circle, i.e., they all point in the horizontal direction. We marked one point $P$ (located at around $150^{\circ}$ on the second-largest ring) in which the students are supposed to identify the direction of the field lines. In the answers of item 3, we did not vary the length of vectors, only focusing on the direction in point $P$. The correct answer is the tangent in point $P$ (pointing roughly in the direction of $60^{\circ}$ ). The distractors include arrows that point radially inward and outward at point $P$ (answers: a, d; students' difficulty: confusion of field lines and equipotential lines [4]); they reflect the horizontal direction that is indicated by the arrows in the field lines (at

3.) The figure below shows a section of a field-line representation. In which direction does the field vector point at point P?

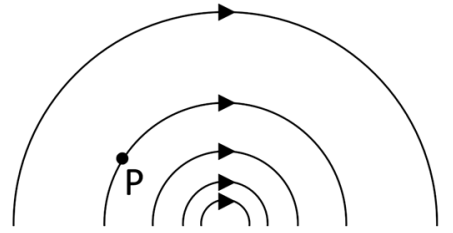

(a)

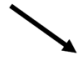

(b)

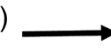

(c) (d)

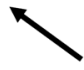

(e)
FIG. 6. Item 3 . 
5.) The figure below shows the vector of a vector field at a point $P$. Indicate for each field line (a) - (c) at point $P$ whether it corresponds to the given vector?

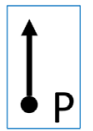

Does the field line correspond to the given vector at point $P$ ?

(a)

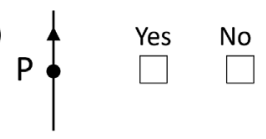

(b)

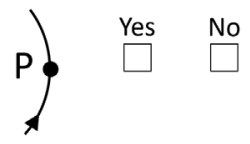

(c)

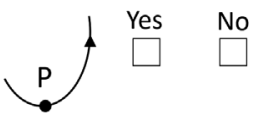

FIG. 7. Item 5.

the topmost point of the circle) in the item stem and a vertical arrow (answers: b, c; students' difficulty: field vectors are not the tangent to field lines [4]).

Item 5 is an item with three true or false questions ( $5 \mathrm{a}$, $5 \mathrm{~b}$, and 5c). It shows a vertical vector at a single point $P$ in the item stem and single field lines in each question. Each question asks whether this field line may correspond to the vector given in the item stem. Question 5a shows a vertical field line in point $P$, which is a correct field-line representation of the given vector and also has surface similarity to the given vector. Question $5 \mathrm{~b}$ shows a curved field line but shows that point $P$ is indicated at a point where the tangent to the field line is also vertical (students' difficulty: preference of a homogeneous solution [56] and field lines must not bend, as inferred from students' interviews). Therefore, the field line in question $5 \mathrm{~b}$ may also correspond to the given vector. Here, the arrow indicating the direction of the vector field is located at a point where the tangent is not vertical. The field line in question $5 \mathrm{c}$ is also curved, and point $P$ is located at a position where the tangent is horizontal. Therefore, $5 \mathrm{c}$ does not correspond to the given vector. In this question, the arrow indicating the direction of the vector field is located at a point where the tangent is vertical (students' difficulty: field vectors are not tangent to field lines [4]). With this systematic variation of the curvature, position of point $P$, and location of the arrow pointing in the direction of the field line, we intended to identify whether the students understand that the vector is the tangent at point $P$ and that the arrow of the field line indicating the direction is not constant but needs to be translated along the line to know about the local direction at point $P$.

In item 4 and item 6 , the students are required to translate the direction of an entire vector field from a vector-field plot to a field-line representation (item 4, Fig. 8) or vice versa (item 6, Fig. 9). Item 4 consists of four true or false questions (4a, 4b, 4c, and 4d), however, subitem $4 \mathrm{~d}$ had low item statistics and has not considered as a part of the test. Item 4 shows a circular vector-field plot with a decreasing magnitude with increasing distance to the center in the item

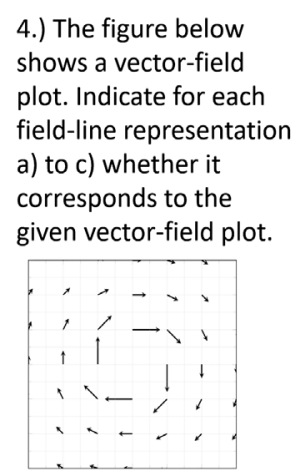

Does the field-line representation correspond to the vector-field plot?

(a)

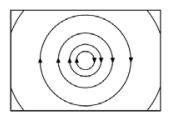

$\begin{array}{ll}\text { Yes } & \text { No } \\ \square \quad \square\end{array}$

(b)
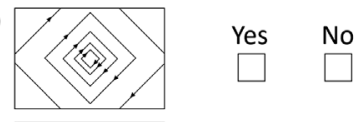

(c)

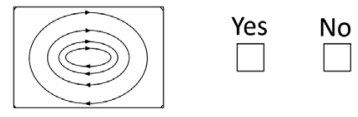

FIG. 8. Item 4.

6.) The figure on the right shows a section of a field-line representation of a vector field. Which vector-field plot corresponds to the field-line representation? (a)

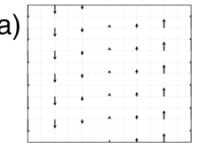

(d)

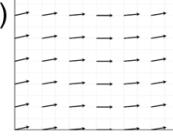

(b)

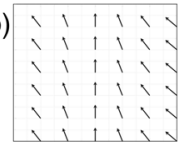

(e)

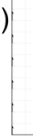

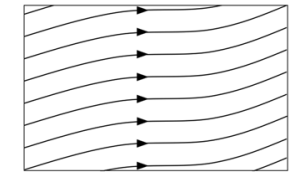

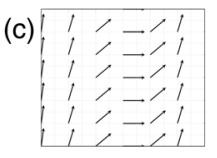

(f) .

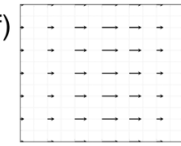

FIG. 9. Item 6 .

stem and field-line representations with different symmetry in each question. Each question asks whether the presented field line may correspond to the vector-field plot given in the item stem. The field lines in each question also have a decreasing magnitude with increasing distance to the center. Therefore, all true or false questions in item 4 mainly address the ability to translate the direction including the symmetry of an entire vector-field plot to a field-line representation. The field-line representation in question $4 \mathrm{a}$ also has circular symmetry and therefore corresponds to the given vector-field plot in the item stem. The arrows of the field lines indicating the direction of the vector field are located along the horizontal line through the center, which means that they point all upward or downward. There are no arrows along the vertical axis through the center, which would cause a higher surface similarity to the vector-field plot in the item stem. The field lines in question $4 \mathrm{~b}$ have a squarelike shape. Therefore, the corners include nondifferentiable points, which imply that no tangents can be determined at these points, and therefore, this field line depiction violates the conventions of field lines. It addresses the students' misunderstanding that field lines may make sharp bends [56]. The field-line representation in question $4 \mathrm{c}$ has an ellipsoidal shape. Therefore, the 
tangents at points along the diagonal lines through the center do not match the directions given by the vectors at points along diagonal lines in the vector-field plot in the item stem. This true or false items includes the previously reported students' difficulty that field lines are not tangent to a field vectors [4]. Identifying this small but relevant quantitative detail of the direction of vectors requires a close inspection of the given vector-field plot. Potentially, students may guess the answer by superficially interpreting the symmetry of the given vector-field plot.

Item 6 contains a field-line representation of homogeneous vector fields with mild changes in direction in the item stem. In this field-line representation, the arrows all point in the horizontal direction. The correct answer consists of a vector-field plot with vectors that represent the tangents to the field lines and exhibit all the same length (answer d). One distractor also follows the same trend, but the change in direction is more exaggerated (distractor $c$ ). Distractor $b$ shows a vector-field plot in which the vectors are all perpendicular to the tangents of the field lines, and therefore it refers to the students' difficulty of the confusion of field lines and equipotential lines [4]. In distractor $\mathrm{f}$, the arrows only point in a horizontal direction, indicating only the changes of the horizontal component of the tangential vectors. Distractor e only points in a vertical direction, indicating only the changes of the vertical component of the tangential vectors. Distractor a shows arrows that point downward and upward indicating the vertical distance of the field lines from an imaginary horizontal line. Therefore, the distractors $\mathrm{a}$, e, and $\mathrm{f}$ all include a variation of the students' difficulty that field vectors are not the tangent to field lines [4]. To solve this item, students need to inspect the directional changes in the center and on the sides in the answer alternatives.

In item 7 and item 8, students need to relate the magnitude of an entire vector field in the field-line representation to a vector-field plot (item 7, Fig. 10) or vice versa (item 8, Fig. 11). Item 7 contains the field-line representation with the change in magnitude in a horizontal

7.) The figure on the right shows the fieldline representation of a vector field. Which vector-field plot corresponds to this fieldline representation? (a)

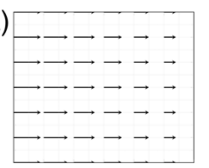

(c)

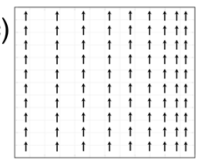

(b)

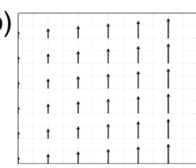

(d)

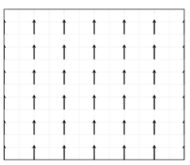

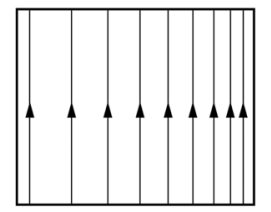

FIG. 10. Item 7.
8.) The figure on the right shows the vectorfield plot of a vector field. Which field-line representation corresponds to this vectorfield plot? (a)

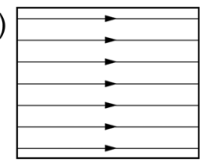

(c)

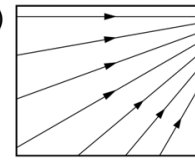

(b)

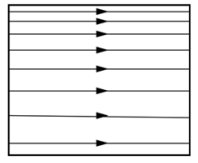

(d)

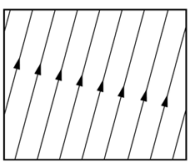

(e)

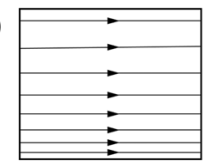

(f)

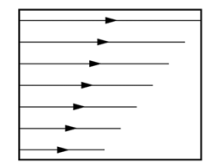

FIG. 11. Item 8.

direction and all field lines pointing upward. The correct vector-field plot (answer b) shows vectors located at equal distances that all point upward and that exhibit an increasing length in a horizontal direction. One alternate answer shows a vector-field plot with vectors all pointing in a vertical direction with an opposite change in length (distractor a). In this distractor, the lengths of the vectors reflect the distances between the field lines in the item stem. The alternate answer e also shows a vector-field plot with all vectors pointing in the opposite vertical direction with an opposite change of magnitude (students' difficulty: confusion of field lines and equipotential lines [4]). Therefore, the distractors a and e are also related to the students' difficulty the field vectors are not tangent to field lines [4]. Distractor c shows a homogeneous vector field of vectors all pointing upward but with the change in distance between vectors resembling the distance between field lines in the item stem (students' difficulty: density represents magnitude as well [4]). Another distractor shows a homogeneous vector field of vectors all pointing upward and all exhibiting the same distance. This means that this distractor shows no change at all.

Item 8 shows a vector-field plot in the item stem with vectors pointing in a horizontal direction and a magnitude change in vertical direction. Accordingly, the correct answer shows a field-line representation with arrows pointing in a horizontal direction with a change in distance between the field lines in the vertical direction (answer alternative b). Distractor e shows a field-line representation with arrows pointing in the horizontal direction but with a change in distance between the field lines in the other vertical direction (students' difficulty: density does not represent magnitude correctly [4]). Distractor a shows the homogeneous vector field with field lines pointing in the same horizontal direction as in the vector-field plot in the item stem (students' difficulty: preference of the homogeneous solution [56]). Distractor $\mathrm{f}$ shows a homogeneous vector field with field lines pointing in the horizontal direction but the change in length of the field lines reflect 
the change in length of the vectors in the vector-field plot (students' difficulty: field lines do not start or end at correct locations [4] and the length of FLs matter, as indicated in the students' interviews). However, this implies that the field lines suddenly end in this representation, which is not in agreement with the convention of field lines. Distractor c shows field lines that all point in the top right corner, which implies that there is a change in magnitude from bottom left to top right and also change in direction among the field lines in this alternate answer (students' difficulty: field lines do not start or end at correct locations [4]). Distractor d shows a homogeneous vector field with arrows pointing along the end points of the vectors in the vector-field plot in the item stem (students' difficulty: field lines are not tangent to field vectors). This means that this field-line representation exhibits a surface similarity to the vectorfield plot in the item stem.

Item 9 and item 10 contain two representations of each type in the item stem, and the task is to superimpose these two vector-field representations (Figs. 12 and 13). Specifically, each of the two items displays two antiparallel homogeneous vector fields with different magnitudes to be superimposed, and therefore, these questions mainly address the understanding of the magnitude of field lines (item 9) and vector-field plots (item 10). In these two items, the distractors have been designed parallel. The correct answer $(9 \mathrm{~d}, 10 \mathrm{f})$ shows the vector field that points in the same direction as the strong vector field in the item stem but with a weaker magnitude. Another distractor $(9 b, 10 d)$ in these two items shows am empty vector field, which would imply that the two vector fields in the item stem cancel each other out (students' difficulties: density does not represent magnitude correctly (9b) [4] and difficulties related to addition of vectors (10d) [4]). Two other distractors (9a,d; $10 \mathrm{c}, \mathrm{e})$ are an exact copy of either the weak vector field in the item stem or the strong vector field in the item stem (students' difficulties: Incomplete direction or magnitude (9a,d) [4], and difficulties related to addition of vectors $(10 \mathrm{c}, \mathrm{e})[4]$, and the arrow length does not match the magnitude of the field $(10 \mathrm{c}, \mathrm{e})$ [4]). There is one distractor (9f,10a) that shows field lines or vectors with alternating

9.) The figures below show the field-line representations of two vector fields. Which field-line representation would be created if the two vector fields are superimposed so that the frames of the two fields exactly overlap?
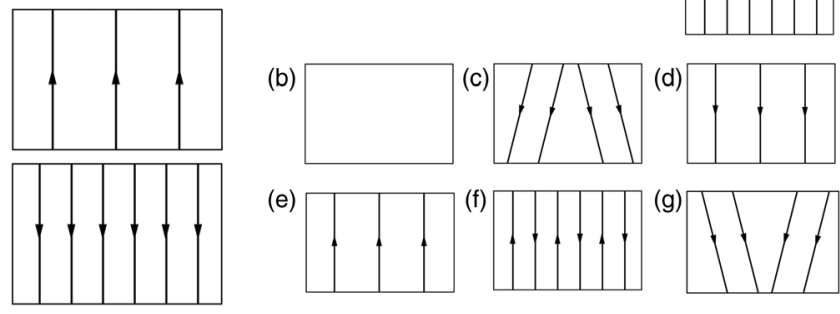

FIG. 12. Item 9.
10.) The figures below show the vector-field plots of two vector fields. Which vector-field plot would be created if the two vector fields are superimposed so that the frames of the two fields exactly overlap?

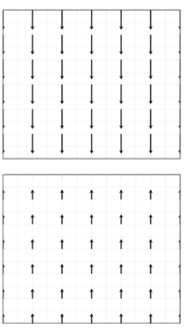

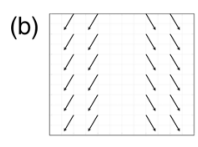

(e)
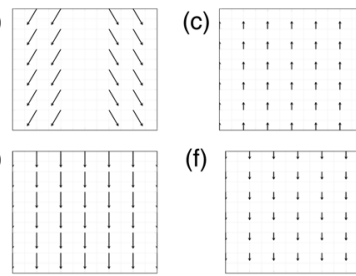

(f)

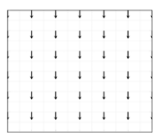

FIG. 13. Item 10 (a)

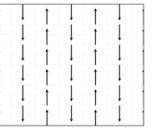

(d)

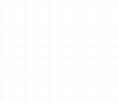

(g)

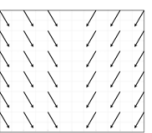

irections among the alternate answers in these two items (students' difficulties: Field is zero in between lines (9a) and vectors (10a) [4]. These alternate answers imply that one would simply overlap the field lines as if there were images and do not interact. Apart from this, two distractors $(9 \mathrm{c}, \mathrm{g} ; 10 \mathrm{~b}, \mathrm{~g})$ show an increased magnitude from top to bottom or from bottom to top (students' difficulty: Difficulties related to addition of vectors $(10 \mathrm{~b}, \mathrm{~g})$ [4]). These two alternate answers may imply that the superposition of the two vector fields in the item stem may cause a deflection of field lines because they collide.

In item 11 and item 12, it is also necessary to superimpose two field-line representations (item 11, Fig. 14) and two vector-field plots (item 12, Fig. 15), but in this case, one of the two vector fields is a circular vector field, and therefore, it is required to understand the influence of the superposition on the magnitude and the direction. Also, in these two items, the answer alternatives have been designed parallel. The correct answer (11e, 12a) in each case shows a pattern that has an enhanced magnitude on the right-hand side of the center and a lower magnitude on the left-hand side. Apart from this, there is also a change in direction on the left-hand side. One distractor $(11 \mathrm{~b}, 12 \mathrm{~d})$ shows the same vector field on the right-hand side but shows an empty area on the left-hand side implying that the two vector

11.) The figures below show the field-line representations of two vector fields. Which field-line representation would be created if the two vector fields are superimposed so that the frames of the two fields exactly overlap?
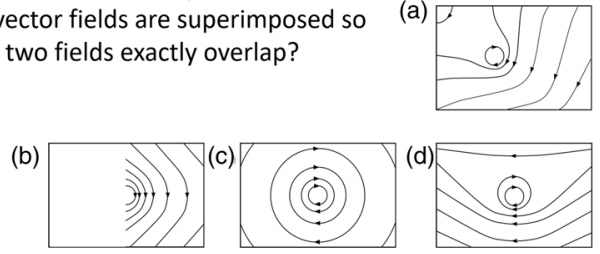

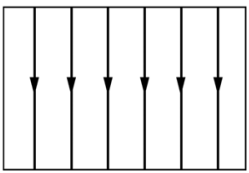

(a)

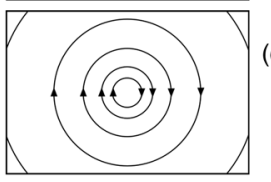

(e)
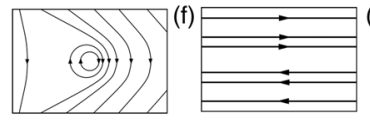
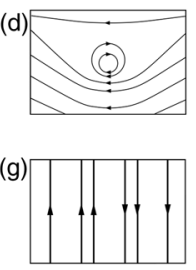

FIG. 14. Item 11. 


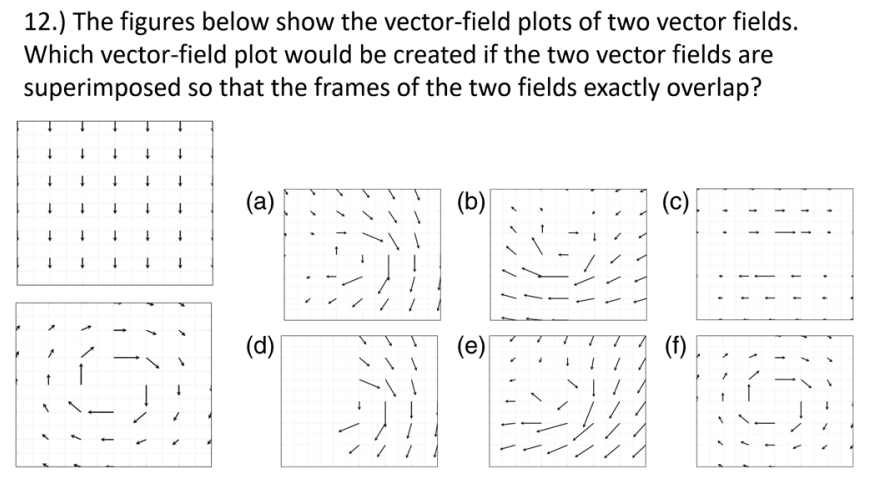

FIG. 15. Item 12.

fields in the item stem cancel each other out on the left-hand side from the center (students' difficulties: field lines do not start or end at the correct locations (11b) [4], incomplete direction or magnitude (11b, 12d) [4], and difficulties related to additional vectors (12d) [4]). There is one distractor $(11 \mathrm{c}, 12 \mathrm{f})$ that is a copy of the circular vector field in the item stem (students' difficulties: incomplete direction or magnitude (11c, 12f) [4]). Another distractor $(11 \mathrm{~d}, 12 \mathrm{~b})$ shows the correct answer but rotated around $90^{\circ}$ in a clockwise direction (students' difficulties: field lines do not start or end at the correct locations (11d) [4], incomplete direction or magnitude (11d, 12b) [4], and difficulties related to the addition of vectors (12b) [4]). Apart from this, one distractor $(11 \mathrm{a}, 12 \mathrm{e})$ shows the result of the superposition of the circular vector field and the homogeneous vector field but with field lines pointing in a diagonal direction (students' difficulties: field lines do not start or end at the correct locations (11a) [4], incomplete direction or magnitude (11a, 12e) [4], and difficulties related to the addition of vectors (12e) [4]). This means that this answer in the two items is slightly skewed with respect to the correct field-line representation, i.e., it does not match with the directions of the field lines of the correct answer. Apart from this, we also included an answer (11f, 12c) that only shows horizontal vectors or field lines with opposite directions on each side of the center (students' difficulties: incomplete direction or magnitude (11f, 12c) [4]). This answer could be chosen if students assume that the vector-field components in vertical directions cancel each other out. In item 11, answer alternative g shows field lines pointing in opposite vertical directions on each side of the center (students' difficulty: incomplete direction or magnitude (11g) [4]). Students might select this answer if they ignore the vertical components of the circular vector field in the item stem entirely, which may happen because there are no arrows on the field lines at these specific points. Of course, this may only happen in the field-line representations and not in the vector-field plots, and therefore, we included this distractor only in item 11 and not in item 12 .
TABLE VIII. Item statistics: Item difficulty index $P_{j}$, item discriminatory index $D_{j}$, and item-test correlation coefficient $r_{j t}$ for items 4 and 5 .

\begin{tabular}{lccc}
\hline \hline Item No. & $P_{j}$ & $D_{j}$ & $r_{j t}$ \\
\hline $4 \mathrm{a}$ & 0.74 & 0.01 & 0.16 \\
$4 \mathrm{~b}$ & 0.73 & 0.17 & 0.29 \\
$4 \mathrm{c}$ & 0.65 & 0.22 & 0.33 \\
$5 \mathrm{a}$ & 0.92 & -0.08 & 0.14 \\
$5 \mathrm{~b}$ & 0.39 & 0.47 & 0.48 \\
$5 \mathrm{c}$ & 0.75 & 0.12 & 0.24 \\
\hline \hline
\end{tabular}

\section{APPENDIX B: DETAILED RESULTS OF ITEMS 4} AND 5 WITHIN THE CLASSICAL TEST THEORY

In the analysis above, we have considered in item 4 and item 5 as correct if all subitems a, b, c were correct and have analyzed the items accordingly. However, for the interested reader, we show the detailed results of the subitems in this section as if they would be treated as separate items within the classical test theory.

\section{APPENDIX C: STUDENT INTERVIEWS}

\section{Item 1:}

In item 1, the students needed to identify the field-line representation with the highest magnitude. An example of an incorrect answer is provided by the following student:

Paul: I would say D, because the field lines-oh, but the arrow lengths are also interesting, I think. Well, I am not sure if it is either the arrow length or the density or it depends on both, so I would vary between A and D, but in $D$ it seems that there is more going on, so I would choose D now.

Here, Paul was undecided about the magnitude convention of vector-field plots and varied between the length of the arrows and the density. Eventually, it seems that he selected answer D because it is more salient.

John: $D$ is the strongest because we see several vector lines or several vectors there and because we have more lines we think there is more force. Where the lines are far apart we assume that the magnitude is lower.

Interviewer: And the arrow length, does it have anything to do with that?

John: Oh, right, look at this. Yes, of course the length of the arrows has something to do with it. Usually with vectors it is like that, the arrow's length of course also says something about the size. I have now assumed that the amount of arrows is... yes well, if we assume the arrow length... then $A$ is the strongest and $D$ the smallest, because D contains the arrows with the shortest length, if we assume that the arrow length really shows the vectors and represents the force. 
Interviewer: It is up to you, either arrow length or density. I mention it only because you did not comment on it.

John: Well, it is possible that $C$ and $B$ are now also simply stronger because the relation between arrow length and distance is exact or perhaps they are all the same size, because the arrow length.... Okay, $C$ and $B$ are different just in direction but they are approximately equal. Yes, they are the same. D has one box that is diagonal, while A has two boxes diagonal. I would actually stick to the fact that $D$ is the stronger one, there is a lower force of the single components, but the density is large enough to say that the general force is stronger.

First, John was rather clear about his decision with answer D. After a question of the interviewer regarding the length of the vectors, John eventually reasoned with a combination of length and vector density.

In the following, Michael considered the context during his comments to his answer to item 1 and eventually ended up with the correct answer.

Michael: D.

Interviewer: Why?

Michael: Most arrows are in one direction.

Interviewer: Does the length have anything to do with it?

Michael: Actually yes, it does. The length of the arrows, the amount, always shows how strong it is. If we had a parallelogram of forces like this, we would have it too. You can also read the strength of the force from it. In this case, it is A because the arrows are the longest.

Similarly to John, Michael first decided on answer D. When asked about the role of the length of a vectors, Michael remembered an example from Newton's mechanics and then eventually decided on the correct answer. This means that the context helped Michael here to decide on the correct answer.

In the following case, David also selected the right answer, but he did not apply any context.

David: So, in my opinion, it is A. I am not quite sure anymore. Once there were vector-field plots and once field lines. For field lines, the strongest vector field is where the lines are closest to each other, and for vectorfield plots, it is where the arrows are longest, and that would be A.

In this case, it is noticeable that David compared the conventions of vector-field plots and field lines during his reasoning and deductively concluded on the correct answer A.

Item 4:

In the following, Michael provided a correct answer and a correct explanation.
Michael: A corresponds to the vector representation, $B$ does not, $C$ also does not, and $D$ does correspond to it. $A$ and $D$ could both be.

Interviewer: Why? The explanation is also important. Michael: Yes, we have concentric circles. So, in answer $B$, it is just like a square. I have never seen that before. With electric field lines and magnetic field lines and there was never such a picture. With answer $C$ it is rather oval, so it is not. But $A$ and $D$ look very concentric. Although no, it cannot be D either. It can only be A. Because with D you can see that the field lines must never be connected, and with $D$, this is not the case.

Here, Michael drew upon his memory to exclude answer B. He also excluded answer $\mathrm{C}$ because of symmetries, and the student remembered that field lines always need to be connected, and therefore, he excluded answer D. Here, the student arrived at the correct answer by referring to one aspect of the convention of field lines, i.e., that field lines cannot intersect in order to be well defined in every point.

In the following example, David arrived at the correct answer by implying an aspect of force vectors.

David: It cannot be answer D because the arrows in the middle are longer than the arrows on the outside, and according to the statement from, before the field lines should be closer together. Then, they are single points. The arrow is always valid for the force, which acts where the arrow starts, so if you draw the arrows, it should not be C; that would not make sense because of the shape. I think it is A. I think it makes circles, so yes. Because of the circular shape.

In this example answer, it is noticeable that David was well aware of several aspects of the convention of field lines and arrived at the correct answer.

\section{Item 5:}

In item 5 , it is necessary to relate the field lines to the vector in a single point. It also consists of three true or false questions. Similar to item 4, when answering to item 5, students typically answer in the way that they mentioned those field lines, which correspond to the vector.

Thomas: Yes, I would also say that A is correct, and B and $C$ are not because a straight vector cannot create round field lines.

In this example, Thomas confused the convention of field lines, which can follow a curved path with the convention of vectors, which are only straight. Therefore, the student correctly chose field-line representation A and falsely excluded field-line representation B.

David: So, it is definitely not $C$ because at point $P$, the tangent of the field line would go to the right and the arrow goes up. Exactly with the reason it would have to 
be $B$ and $A$ would have to be correct. $A$ is very obvious; you can transfer it almost one to one, but at $B$ it is a circle and the tangent of the circle at point $P$ is perpendicular at point $P$, so this should fit.

In this example, David correctly translated information from field-line representations to vectors by using the concept of a tangent at a point and arrived at the correct answer.

Item 7:

Item 7 is a single-choice question, which showed a fieldline representation with a change in magnitude, and the task consisted in identifying the corresponding vector-field plot.

Michael: I would say C, because it looks most similar. Yes and the arrows are all on the same level.

The answer of Michael is a typical example of the answers of students who selected answer C. Here, Michael derived his answer from surface similarities of the vectorfield plot representation and the field-line representation.

Robert: Well, I think A is right. So because of the direction of the vectors. How should I explain this? First because of the size. There is a difference.

Interviewer: Do you mean the length?

Robert: The difference between the length, I think, and the direction-the vectors should be horizontal.

Interviewer: Even if the arrows in the picture are pointing upwards?

Robert: Yes. So orthogonal.

In this example, Robert assumed an orthogonal relationship between the vectors and the field lines. The student selected the incorrect answer A because, in his opinion, the length of the vectors are related to the vertical distance of the field lines.

In the following correct answer of David, he realized that the density of field lines is related to the magnitude and identified the same metric with the length of the vectors in the vector-field plot.

David: It is not $A$ and $E$ because the field vectors are going in a different direction than upwards. Then, the field becomes stronger and stronger from left to right. Accordingly, the arrows should become longer, and therefore, it is $B$.

\section{Item 8:}

Item 8 is a single-choice item and the analog question to item 7 for vector-field plots. The item stem shows a vectorfield plot with a change in magnitude, and students needed to identify the corresponding field-line representation.

Robert: I choose F because the vectors below are shorter, so the field lines below should normally be shorter.
Here, Robert chose the incorrect answer alternative $\mathrm{F}$ because there is the surface similarity between the field lines and the vectors in the vector-field plot.

Eric: I would exclude D and C again because they don't match the direction. I would also exclude $F$ because the field lines end in nowhere. I would also exclude A because nothing changes there. Above, we see in the picture of field-lines that the vectors are longer at the top and shorter at the bottom. In answer A they would all be the same. I would say $B$ is correct and corresponds to the vector image because the field lines are denser at the top and less dense at the bottom, which would correspond to the length of the vector arrows. Answer E, I would say, does not match because it is the wrong way around.

Here, Eric correctly related the magnitude convention of vector-field plots and field lines and arrived at the correct answer B.

Item 9:

Item 9 is a single-choice question, and it shows two fieldline representations of homogeneous vector fields in the item stem. The task is to find the field-line representation that corresponds to the superposition of these two vector fields.

John: So $G$ and $C$ make no sense. We have a pure vertical direction from the field lines, which means we will never have a distraction, I would say. That's why I would simply exclude them. So, the question is now, if the field lines had the same magnitude or, the vectors, if we assume that the same size corresponds to the same value, then we would have three arrows pointing down in $D$, then three ones would cancel each other, let's say, and then $D$ would be correct in any case. E would then make no sense, unless we had a greater force from the field lines pointing upward. And F does not make any sense either because we have only three lines going down and three lines going up, and we already have six lines going down.

Interviewer: Okay, is there a way to tell if there is a greater field at the top or at the bottom?

John: Maybe by the length of the line, that might be possible. Or by the distance between the lines, I already had that. Oh yes, of course, it could be recognized by the distance between the lines.

Interviewer: Okay, what does this mean then?

John: It could be that it is also E. But E doesn't make any sense either, because the lines there, that is .... Oh, B is empty; I didn't even notice that. So, an empty field would theoretically also be possible that they completely erase each other. Yes, that could also be. I would now commit myself to $B$ because I think that just by the field-line 
distances below the total force could be exactly the same as above the total force.

In this example, John correctly excluded alternatives $C$ and $\mathrm{G}$ because the vector fields, which need to be superimposed in this item do not have any component in horizontal direction. Then, he simply added the field lines one by one but eventually chose answer B because he argued that the magnitudes in both vector fields are the same.

David: I would exclude $G$ and $C$ because the field lines are only directed up and down, and therefore, it could not go into the slope. F makes no sense, in my opinion, because I want to have a resulting field, and the field lines in this case all compensate each other, so all arrows must go in one direction. B also makes no sense because the lower field is much stronger than the upper field, i.e., the resulting field must also go down, but it must also be weaker, i.e., less dense than the original image. Accordingly, it should be D.

In this answer, David correctly realized that the field with field lines pointing downward has a higher magnitude than the vector field with field lines pointing upward because the field lines are denser. Thus, he chose the correct answer D in which the resulting vector field after superposition is weaker and pointing downward.

Item 10:

Item 10 is a single-choice item, which is the analog item to question 9 with the task to superimpose two homogeneous vector-field plots.

Kevin: I would choose D because they are opposite and they cancel each other out. It seems that Kevin only considered the direction of both vector fields and incorrectly chose answer $D$ as both vector fields cancel each other out.

David: With the same reasoning as before, $A, B$, and $G$ do not work either. (Reason before: See answer of David in item 9). So, the first reason. First, the orientation of the field can only be up or down. In this case, it must be downwards because the upper field is stronger than the lower one, which can be seen by the longer arrows. Accordingly, it cannot be D, of course. E would be the same picture as the strong field, but it is obviously weakened by the weaker field, which is the opposite. So, it should be F.

In this correct answer, David first considered the direction and excluded answers $\mathrm{B}$ and $\mathrm{G}$, and then he focused on the magnitude of both vector fields by looking at the length of the arrows.

Item 11:

In this item, the students needed to superimpose two field-line representations of a homogeneous linear vector field and a circular vector field with decreasing magnitude with increasing distance from the center.
Mark: I am torn between $E$ and A, but I am actually tending more to $E$ than to $A$. Because in the first picture are the lines, at least this time, I am sure that it is the same distance and probably implies that it is the same magnitude value of the field lines. In the lower picture, the distance between the field lines is getting bigger and bigger, so that the whole thing probably becomes weaker towards the outside. Therefore, I could imagine that as in picture E the complete outside could act again somewhat downward because of the weakness of the field lines from the lower, given picture because there the field lines are only very weak. Accordingly, although it makes no sense, that... yes, it does, and accordingly the picture is warped downwards. Now I'm just wondering if answer A would not make sense in a certain way, since the... actually, A makes more sense. Because there the picture is more distorted downwards by the lines. Because in picture E, the whole thing is again curved relatively strongly to the left in the lower half, but that would have to be curved relatively strongly, more strongly downwards again. Yes, I think it is A.

In this example, Mark first correctly realized that the homogeneous vector field has a constant magnitude as the distance between field lines is constant and the circular vector field is becoming weaker with increasing distance to the center. Then, he varied between answer A and answer E and eventually decided on the incorrect answer A because, in his point of view, in this option, there is a stronger curvature in the lower left quarter.

Kevin: I would choose $G$ because in the second picture, the field lines are running in a circle, and sometimes they are running up and sometimes down. So I would say, if they are opposite, then they cancel each other out, and if they point in the same direction, then they will overlap and are stronger, and therefore, only G fits.

In this incorrect reason, Kevin exclusively considered the superposition of the vertical components of the circular field lines in the field lines of the homogeneous vector field and ignored the horizontal components of the circular field. Consequently, Kevin selected the incorrect answer G.

David: So, on the right half of the resulting field-line picture, the two directions of the field lines support each other. Ergo, the vector field should be especially strong there. At least stronger than on the left side, which would correspond to $A, B$, and $E$. On the other side, they would eliminate each other for a short time and then the vector field would have to go down again. As the closer you go to the center of the circle, the stronger the vector field is at the bottom. Accordingly, it would have to be E.

In this argument, David first considered the "righthand" side from the center in the circular field-line 
representation and realized that the two vector fields in this region constructively overlap. With this observation, he reduced the answer alternatives to option A, B, and E. In the final step of his reasoning, David superimposed the components of each vector field and selected the correct answer E.

\section{Item 12:}

In this final item, the students also had to superimpose a homogeneous linear vector field and the circular vector field with decreasing magnitude with increasing distance from the center. In contrast to item 11, the vector-field plot representations of the two vector fields were given.

Kevin: I would choose E. because of the same reason as in the other task. Because, for example, if the vectors in both illustrations point in the same direction on the right side, then it will be stronger, but those on the left side, some are opposite, and they will then cancel each other out.

In this example, Kevin first correctly observes that the magnitude on the right-hand side of the center is stronger after superposition in comparison to the original vector fields, and then he argues that on the left-hand side some arrows cancel each other out. Although both observations are correct, Kevin selects the incorrect answer alternative E.
David: So, on the right half, there should be long arrows, which are mostly oriented downwards. On the left side there should be short arrows. In between they could vary. So, E it is not. Answer $C$ it is not because of the orientation in space. I would also exclude F because the lower picture must definitely change, and the resulting picture cannot simply be the lower picture, because I put another field on top of it. It cannot be answer $B$ either because just below the center of the circle from the first lower image there is a very long arrow in B just below the center, a rather strong size, which should be oriented downward because the upper field is pressing down. Therefore, I would now answer either A or C. It must be A because if an arrow points down and an arrow points up, the arrows do not point to the left. So, it should be A.

In this correct explanation, David realized that the superimposed vector field needs to consist of longer arrows on the right half and of shorter arrows on the left in comparison to the two initial vector fields. He excluded answers $\mathrm{C}$ and $\mathrm{F}$ because they neglect certain factors. Eventually, David excluded answer B because of the orientation and length of a single vector just below the center and, also, a single vector on the left-hand side from the center allowed the student to discard answer E.
[1] E. Ekici, "Why Do I Slog through the Physics?", Understanding high school students' difficulties in learning physics, J. Educ. Pract. 7, 95 (2016).

[2] S. Ainsworth, DeFT: A conceptual framework for considering learning with multiple representations, Learning Instr. 16, 183 (2006).

[3] E. Campos, G. Zavala, K. Zuza, and J. Guisasola, Students' understanding of the concept of the electric field through conversions of multiple representations, Phys. Rev. Phys. Educ. Res. 16, 010135 (2020).

[4] L. Bollen, P. van Kampen, C. Baily, M. Kelly, and M. De Cock, Student difficulties regarding symbolic and graphical representations of vector fields, Phys. Rev. Phys. Educ. Res. 13, 020109 (2017).

[5] G. Brod, Generative Learning: Which Strategies for what Age?, Educ. Psychol. Rev. 1 (2020).

[6] A. A. diSessa, Metarepresentation: Native Competence and Targets for Instruction, Cognit. Instr. 22, 293 (2004).

[7] R. Even, Factors involved in linking representations of functions, J. Math. Behav. 17, 105 (1998).

[8] J. K. Gilbert and D. Treagust, Towards a coherent model for macro, submicro and symbolic representations in chemical education, in Multiple Representations in Chemical Education, edited by J. K. Gilbert and D. Treagust (Springer, Dordrecht, Netherlands, 2009), pp. 333-350.
[9] A. Van Heuvelen, Learning to think like a physicist: A review of research-based instructional strategies, Am. J. Phys. 59, 891 (1991).

[10] A. Van Heuvelen and X. Zou, Multiple representations of work-energy processes, Am. J. Phys. 69, 184 (2001).

[11] M. A. Rau, V. Aleven, and N. Rummel, Intelligent tutoring systems with multiple representations and self-explanation prompts support learning of fractions, in Proceedings of the 14th International Conference on Artificial Intelligence in Education, edited by V. Dimitrova, R. Mizoguchi, and B. Du Boulay (IOS Press, Amsterdam, 2009), pp. 441-448.

[12] D. Rosengrant, E. Etkina, and A. Van Heuvelen, An overview of recent research on multiple representations, AIP Conf. Proc. 883, 149 (2007).

[13] C. Y. Tsui and D. F. Treagust, Introduction to multiple representations: Their importance in biology and biological education, in Multiple Representations in Biological Education. Models and Modeling in Science Education, edited by D. F. Treagust and C. Y. Tsui (Springer, Dordrecht, 2013), Vol. 7.

[14] Constructing Representations to Learn in Science, edited by R. Tytler, V. Prain, P. Hubber, and B. Waldrip (SensePublishers, Rotterdam, 2013).

[15] P. Klein, J. Viiri, S. Mozaffari, A. Dengel, and J. Kuhn, Instruction-based clinical eye-tracking study on the visual interpretation of divergence: How do students look at 
vector field plots?, Phys. Rev. Phys. Educ. Res. 14, 010116 (2018).

[16] M. Reisslein, R. Moreno, and G. Ozogul, Pre-college electrical engineering instruction: The impact of abstract vs. contextualized representation and practice on learning, J. Eng. Educ. 99, 225 (2010).

[17] W. Schnotz, Integrated model of text and picture comprehension, in The Cambridge Handbook of Multimedia Learning, 2nd ed., edited by R. E. Mayer (Cambridge University Press, Cambridge, England, 2014), pp. 72-103.

[18] T. Fredlund, C. Linder, J. Airey, and A. Linder, Unpacking physics representations: Towards an appreciation of disciplinary affordance, Phys. Rev. ST Phys. Educ. Res. 10, 020129 (2014).

[19] S. Ainsworth, The functions of multiple representations, Comput. Educ. 33, 131 (1999).

[20] R. B. Kozma, J. Russell, T. Jones, N. Marx, and J. Davis, The use of multiple, linked representations to facilitate science understanding, in International Perspectives on the Design of Technology-Supported Learning Environments, edited by S. Vosniadou, E. De Corte, R. Glaser, \& H. Mandl (Lawrence Erlbaum Associates, Inc., Hillsdale, NJ, 1996), pp. 41-60.

[21] R. E. Mayer, The Cambridge Handbook of Multimedia Learning, 2nd ed. (Cambridge University Press, Cambridge, England, 2014).

[22] C. Linder, Disciplinary discourse, representation, and appresentation in the teaching and learning of science, Eur. J. Sci. Math. Educ. 1, 43 (2013).

[23] S. E. Ainsworth, P. A. Bibby, and D. J. Wood, Examining the effects of different multiple representational systems in learning primary mathematics, J. Learn. Sci. 11, 25 (2002).

[24] W.-M. Roth, G. M. Bowen, and M. K. McGinn, Differences in graph-related practices between high school biology textbooks and scientific ecology journals, J. Res. Sci. Teach. 36, 977 (1999).

[25] M. P. Cook, Visual representations in science education: The influence of prior knowledge and cognitive load theory on instructional design principles, Sci. Educ. 90, 1073 (2006).

[26] P. Hubber, R. Tytler, and F. Haslam, Teaching and learning about force with a representational focus: Pedagogy and teacher change, Res. Sci. Educ. 40, 5 (2010).

[27] P. Kohl, D. Rosengrant, and N. Finkelstein, Comparing explicit and implicit teaching of multiple representation use in physics problem solving, AIP Conf. Proc. 883, 145 (2007).

[28] J. Clement, The concept of variation and misconceptions in Cartesian graphing, Focus on Learning Problems Math. 11, 77 (1989).

[29] P. Nieminen, A. Savinainen, and J. Viiri, Relations between representational consistency, conceptual understanding of the force concept, and scientific reasoning. Phys. Rev. ST Phys. Educ. Res. 8, 010123 (2012).

[30] A. A. diSessa and B. L. Sherin, Meta-representation: An introduction, J. Math. Behav. 19, 385 (2000).

[31] R. B. Kozma and J. Russell, Multimedia and understanding: Expert and novice responses to different representations of chemical phenomena, J. Res. Sci. Teach. 34, 949 (1997).
[32] P. Klein, A. Müller, and J. Kuhn, Assessment of representational competence in kinematics, Phys. Rev. Phys. Educ. Res. 13, 010132 (2017).

[33] K. L. Daniel, C. J. Bucklin, E. Austin Leone, and J. Idema, Towards a definition of representational competence, in Towards a Framework for Representational Competence in Science Education. Models and Modeling in Science Education, edited by K. Daniel (Springer, Cham, 2018), Vol. 11.

[34] M. E. Lira and M. Stieff, Using gesture analysis to assess students' developing representational competence, in Towards a Framework for Representational Competence in Science Education. Models and Modeling in Science Education, edited by K. Daniel (Springer, Cham, 2018), Vol. 11.

[35] J. Scheid, A. Müller, R. Hettmannsperger, and W. Schnotz, Improving learners' representational coherence ability with experiment-related representational activity tasks, Phys. Rev. Phys. Educ. Res. 15, 010142 (2019).

[36] R. B. Kozma and J. Russel, Students becoming chemists: developing representational competence, in Visualization in Science Education, edited by J. Gilbert (Kluwer, London, 2005).

[37] M. Niss, M. Prescriptive modelling — challenges and opportunities, in Mathematical Modelling in Education Research and Practice: Cultural, Social and Cognitive Influences, edited by G. A. Stillman, W. Blum, and M. Biembengut (Springer, Cham, 2015), pp. 67-79.

[38] R. Tytler, V. Prain, and S. Peterson, Representational issues in students learning about evaporation, Res. Sci. Educ. 37, 313 (2007).

[39] M. A. Rau, Conditions for the effectiveness of multiple visual representations in enhancing STEM learning, Educ. Psychol. Rev. 29, 717 (2017).

[40] M. A. Rau, Cognitive and socio-cultural theories on competencies and practices involved in learning with multiple external representations, in Handbook of Learning from Multiple Representations and Perspectives, edited by P. Van Meter, A. List, D. Lombardi, and P. Kendeou (Routledge, New York, 2020).

[41] D. E. Meltzer, Relation between students' problem-solving performance and representational format, Am. J. Phys. 73, 463 (2005).

[42] A. Northedge, Organizing excursions Into specialist discourse communities: A sociocultural account of university teaching, in Learning for Life in the 21st Century, edited by G. Wells and G. Claxton (Wiley, New York, 2002).

[43] O. Parnafes, What Does "Fast" Mean? Understanding the Physical World Through Computational Representations, J. Learn. Sci. 16, 415 (2007).

[44] R. J. Beichner, Testing student interpretation of kinematics graphs, Am. J. Phys. 62, 750 (1994).

[45] P. Nieminen, A. Savinainen, and J. Viiri, Force Concept Inventory-based multiple-choice test for investigating students' representational consistency, Phys. Rev. ST Phys. Educ. Res. 6, 020109 (2010).

[46] J. Scheid, A. Müller, R. Hettmannsperger, and J. Kuhn, Erhebung von repräsentationaler Kohärenzfähigkeit von Schülerinnen und Schülern im Themenbereich Strahlenoptik, Z. Didaktik der Natur. 23, 181 (2017). 
[47] E. R. Fyfe, N. M. McNeil, J. Y. Son, and R. L. Goldstone, Concreteness fading in mathematics and science instruction: A systematic review, Educ. Psychol. Rev. 26, 9 (2014).

[48] A. Donhauser, S. Küchemann, J. Kuhn, M. Rau, S. Malone, P. Edelsbrunner, and A. Lichtenberger, Making the invisible visible: Visualization of the connection between magnetic field, electric current, and Lorentz force with the help of augmented reality, Phys. Teach. 58, 438 (2020).

[49] J. Lincoln, Electric field patterns made visible with potassium permanganate, Phys. Teach. 55, 74 (2017).

[50] A. I. Benimoff, The electric fields experiment: A new way using conductive tape, Phys. Teach. 44, 140 (2006).

[51] S. P. Thompson, A study in magnetism, Nature (London) 19, 79 (1878).

[52] D.-H. Nguyen and S. Rebello, Students' difficulties in transfer of problem solving across representations, AIP Conf. Proc. 1179, 221 (2009).

[53] D.-H. Nguyen, E. Gire, and S. Rebello, Facilitating students' problem solving across multiple representations in introductory mechanics, AIP Conf. Proc. 1289, 45 (2010).

[54] S. Van den Eynde, P. van Kampen, W. Van Dooren, and M. De Cock, Translating between graphs and equations: The influence of context, direction of translation, and function type, Phys. Rev. Phys. Educ. Res. 15, 020113 (2019).

[55] E. Gire and E. Price, Arrows as anchors: An analysis of the material features of electric field vector arrows, Phys. Rev. ST Phys. Educ. Res. 10, 020112 (2014).

[56] S. Törnkvist, K.-A. Pettersson, and G. Tranströmer, Confusion by representation: On student's comprehension of the electric field concept, Am. J. Phys. 61, 335 (1993).

[57] D. A. Frisbie and D. C. Sweeney, The relative merits of multiple true-false tests, J. Educ. Measure. 19, 29 (1982).
[58] A. Mobalegh and H. Barati, Multiple True-false (MTF) and multiple-choice (MC) test formats, J. Lang. Teach. Res. 3, 1027 (2012).

[59] B. A. Couch, J. K. Hubbard, and C. E. Brassil, Multipletrue-false questions reveal the limits of the multiple-choice format for detecting students with incomplete understandings, Bio Science 68, 455 (2018).

[60] J. K. Hubbard, M. A. Potts, and B. A. Couch, How question types reveal student thinking: An experimental comparison of multiple-true-false and free-response formats, CBE Life Sci. Educ. 16, ar26 (2017).

[61] https://www.unipark.com/

[62] S. Malone, S. Küchemann, P. A. Edelsbrunner, A. Lichtenberger, K. Altmeyer, R. Schumacher, and R. Brünken, CESAR 0 data. Retrieved from osf.io/p476u (May 5, 2021).

[63] T. Bond, Z. Yan, and M. Heene, Applying the Rasch Model: Fundamental Measurement in the Human Sciences (Routledge, New York, 2020).

[64] R. L. Doran, Basic Measurement and Evaluation of Science Instruction (National Science Teachers Association, Washington, DC, 1980).

[65] L. Ding and R. Beichner, Approaches to data analysis of multiple-choice questions, Phys. Rev. ST Phys. Educ. Res. 5, 020103 (2009).

[66] T. J. Dunn, T. Baguley, and V. Brunsden, From alpha to omega: A practical solution to the pervasive problem of internal consistency estimation, Brit. J. Psychol. 105, 399 (2014).

[67] A. Robitzsch, T. Kiefer, and M. Wu, Package TAM: Test Analysis Modules (2020).

[68] B. Wright, Reasonable mean-square fit values, Rasch Meas Trans. 8, 370 (1994).

[69] L. J. Cronbach and P. E. Meehl, Construct validity in psychological tests, Psychol. Bull. 52, 281 (1955). 